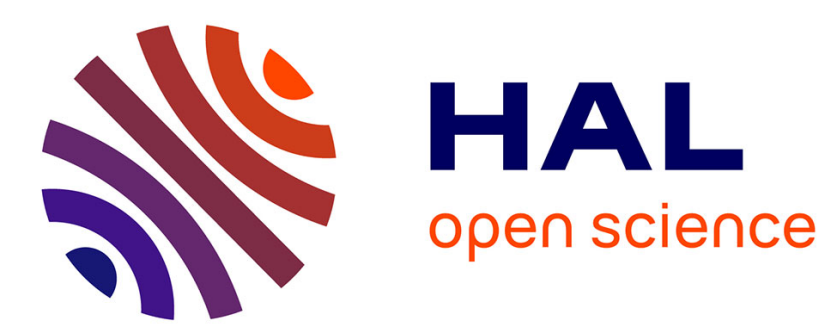

\title{
The finite Larmor radius regime: collisional setting and fluid models
}

\author{
Mihai Bostan, Aurélie Finot
}

\section{To cite this version:}

Mihai Bostan, Aurélie Finot. The finite Larmor radius regime: collisional setting and fluid models. Communications in Contemporary Mathematics, In press, 10.1142/S0219199719500470 . hal01706310v2

\section{HAL Id: hal-01706310 \\ https://hal.science/hal-01706310v2}

Submitted on 19 Aug 2019

HAL is a multi-disciplinary open access archive for the deposit and dissemination of scientific research documents, whether they are published or not. The documents may come from teaching and research institutions in France or abroad, or from public or private research centers.
L'archive ouverte pluridisciplinaire HAL, est destinée au dépôt et à la diffusion de documents scientifiques de niveau recherche, publiés ou non, émanant des établissements d'enseignement et de recherche français ou étrangers, des laboratoires publics ou privés. 


\title{
The finite Larmor radius regime : collisional setting and fluid models
}

\author{
Mihaï BOSTAN *, Aurélie FINOT ${ }^{\dagger}$
}

\begin{abstract}
The subject matter of this paper concerns the derivation of fluid limits for gyro-kinetic models. The arguments apply for any collision kernel satisfying the usual conservations (mass, momentum, kinetic energy) and possessing a production entropy sign. We describe the set of equilibria in terms of several moments, we determine the average collision invariants, and we write the associated macroscopic equations and the entropy inequality.
\end{abstract}

Keywords: Finite Larmor radius regime, Fokker-Planck-Landau equation, Euler equations.

AMS classification: $35 \mathrm{Q} 75,78 \mathrm{~A} 35,82 \mathrm{D} 10$.

\section{Introduction}

Let $f=f(t, x, v)$ be the presence density of a population of charged particles of mass $m$, charge $q$. Motivated by the magnetic confinement, we concentrate on the dynamics of this population in presence of a strong magnetic field, by taking into account the collision effects $[14,15,16,22,25]$. The particles are transported under the action of the electro-magnetic force $q(E+v \wedge B)$, and their density fluctuates also due to the collision mechanism

$$
\partial_{t} f+v \cdot \nabla_{x} f+\frac{q}{m}(E+v \wedge B) \cdot \nabla_{v} f=Q(f), \quad(t, x, v) \in \mathbb{R}_{+} \times \mathbb{R}^{3} \times \mathbb{R}^{3} .
$$

We supplement the above equation by the initial condition

$$
f(0, x, v)=f^{\text {in }}(x, v), \quad(x, v) \in \mathbb{R}^{3} \times \mathbb{R}^{3} .
$$

*Aix Marseille Université, CNRS, Centrale Marseille, I2M, Marseille France, Centre de Mathématiques et Informatique, UMR 7373, 39 rue Frédéric Joliot Curie, 13453 Marseille Cedex 13 France. E-mail : mihai.bostan@univ-amu.fr

†Aix Marseille Université, CNRS, Centrale Marseille, I2M, Marseille France, Centre de Mathématiques et Informatique, UMR 7373, 39 rue Frédéric Joliot Curie, 13453 Marseille Cedex 13 France. E-mail : aurelie.finot@univ-amu.fr. 
The appropriate collision operator when studying the magnetic confinement is given by the Fokker-Planck-Landau kernel

$$
Q_{F P L}(f)=\operatorname{div}_{v} \int_{\mathbb{R}^{3}} \sigma\left(\left|v-v^{\prime}\right|\right)\left|v-v^{\prime}\right|^{3} S\left(v-v^{\prime}\right)\left(f\left(v^{\prime}\right)(\nabla f)(v)-f(v)(\nabla f)\left(v^{\prime}\right)\right) \mathrm{d} v^{\prime}
$$

where $\sigma>0$ is the scattering cross section, and $S(w)=I_{3}-w \otimes w /|w|^{2}$ represents the orthogonal projection on the plane perpendicular to $w \in \mathbb{R}^{3} \backslash\{0\}$. The computation of the effective collision kernel, under strong magnetic fields has been done for both linear (Boltzmann, Fokker-Planck) and non linear ( Fokker-Planck-Landau, Boltzmann) operators $[5,7,8,9,10,13]$. In these works, completely explicit expressions for the effective collision kernels have been emphasized. In particular, their equilibria were identified and fluid models were derived, by linearizing around these configurations. Nevertheless, these derivations require a huge amount of computations. Here we propose a different approach which allows us to identify the equilibria of the average collision kernels, without having to compute explicitly the effective collision kernels. The main point is to observe that the effective kernels still satisfy a set of balances, and that a $H$ Theorem is available as well. We concentrate on the finite Larmor radius regime. For simplicity we suppose that the magnetic field is uniform $\mathbf{B}={ }^{t}(0,0, B)$. We take into account the collision effects as well. More exactly, we distinguish between

- the perpendicular dynamics, generated by

$$
b(x, v) \cdot \nabla_{x, v}=v_{1} \partial_{x_{1}}+v_{2} \partial_{x_{2}}+\omega\left(v_{2} \partial_{v_{1}}-v_{1} \partial_{v_{2}}\right), \quad \omega=\frac{q B}{m}
$$

- the collision mechanism, given by the Fokker-Planck-Landau kernel

- the parallel dynamics, generated by

$$
a(t, x, v) \cdot \nabla_{x, v}=v_{3} \partial_{x_{3}}+\frac{q}{m} E(t, x) \cdot \nabla_{v} .
$$

We assume that the perpendicular dynamics evolves on a much smaller time scale than the typical collision time, and that the collision time is negligible with respect to the time scale on which evolves the parallel dynamics

$$
T_{\perp}<<T_{\text {coll }}<<T_{\|}
$$

This asymptotic regime is characterized by two small parameters $\varepsilon>0, \tau>0$ which represent the ratio $\frac{T_{\perp}}{T_{\text {coll }}}$ and $\frac{T_{\text {coll }}}{T_{\|}}$. We intend to analyze the behavior of the family $\left(f^{\varepsilon, \tau}\right)_{\varepsilon, \tau>0}$ when first $\varepsilon=\frac{T_{\perp}}{T_{\text {coll }}} \rightarrow 0$ and then $\tau=\frac{T_{\text {coll }}}{T_{\|}} \rightarrow 0$. We appeal to average methods $[3,4,6,11,12,21]$ and we derive a fluid gyro-kinetic limit, at least at the formal level, for the problems

$$
\begin{gathered}
\partial_{t} f^{\varepsilon, \tau}+a(t, x, v) \cdot \nabla_{x, v} f^{\varepsilon, \tau}+\frac{b(x, v)}{\varepsilon} \cdot \nabla_{x, v} f^{\varepsilon, \tau}=\frac{1}{\tau} \tilde{Q}_{F P L}\left(f^{\varepsilon, \tau}(t)\right), \quad(t, x, v) \in \mathbb{R}_{+} \times \mathbb{R}^{3} \times \mathbb{R}^{3} \\
f^{\varepsilon, \tau}(0, x, v)=f^{\text {in }}(x, v), \quad(x, v) \in \mathbb{R}^{3} \times \mathbb{R}^{3}
\end{gathered}
$$


where for any presence density $g=g(x, v)$, the notation $\tilde{Q}_{F P L}(g)(x, v)$ stands for $Q_{F P L}(g(x, \cdot))(v)$.

The perpendicular dynamics is given by $(\mathcal{X}, \mathcal{V})(s=t / \varepsilon ; x, v)$, where $\mathcal{Z}=(\mathcal{X}, \mathcal{V})$ is the characteristic flow associated to the vector field $b \cdot \nabla_{x, v}$

$$
\frac{\mathrm{d} \mathcal{X}}{\mathrm{d} s}={ }^{t}\left(\mathcal{V}_{1}(s ; x, v), \mathcal{V}_{2}(s ; x, v), 0\right), \frac{\mathrm{d} \mathcal{V}}{\mathrm{d} s}=\omega^{t}\left(\mathcal{V}_{2}(s ; x, v),-\mathcal{V}_{1}(s ; x, v), 0\right)
$$

with $(\mathcal{X}, \mathcal{V})(0 ; x, v)=(x, v)$. This flow, denoted by $\mathcal{Z}(s ; z)=(\mathcal{X}(s ; x, v), \mathcal{V}(s ; x, v))$, $z=(x, v)$, is $T_{c}=2 \pi / \omega$ periodic, and writes

$$
\mathcal{X}(s ; x, v)=\left(\bar{x}+\omega^{-1}\left(I_{2}-\mathcal{R}(-\omega s)\right)^{\perp} \bar{v}, x_{3}\right), \quad \mathcal{V}(s ; x, v)=\left(\mathcal{R}(-\omega s) \bar{v}, v_{3}\right)
$$

where we have used the notations $\bar{x}=\left(x_{1}, x_{2}\right), \bar{v}=\left(v_{1}, v_{2}\right),{ }^{\perp} \bar{v}=\left(v_{2},-v_{1}\right)$. We denote by $\mathcal{R}(\theta)$ the rotation of angle $\theta \in \mathbb{R}$ in $\mathbb{R}^{2}$. The above expressions come easily, by observing that $\bar{x}+{ }^{\perp} \bar{v} / \omega, x_{3},|\bar{v}|, v_{3}$ are left invariant by the flow $(\mathcal{X}, \mathcal{V})$. In particular, the Larmor center $\bar{x}+{ }^{\perp} \bar{v} / \omega$ and the Larmor radius $|\bar{v}| /|\omega|$ are conserved by the perpendicular dynamics, whose time scale is given by the cyclotronic period $T_{c}^{\varepsilon}=\varepsilon T_{c}=\varepsilon \frac{2 \pi}{\omega}=\frac{2 \pi m}{q B / \varepsilon}$.

We proceed by smoothing out the oscillations due to the fast cyclotronic motion. More exactly, we search for a new family of presence densities such that at any time $t$, $f^{\varepsilon, \tau}(t)$ appears as the composition between $F^{\varepsilon, \tau}(t)$ and the fast oscillating characteristic flow of $\frac{b}{\varepsilon} \cdot \nabla_{x, v}$

$$
f^{\varepsilon, \tau}(t, x, v)=F^{\varepsilon, \tau}(t, X, V), \quad \text { with } Z=(X, V)=(\mathcal{X}, \mathcal{V})(-t / \varepsilon ; x, v) .
$$

It is easily seen that

$$
\partial_{t} F^{\varepsilon, \tau}(t, Z)=\partial_{t} f^{\varepsilon, \tau}(t, \mathcal{Z}(t / \varepsilon ; Z))+\frac{b}{\varepsilon}(\mathcal{Z}(t / \varepsilon ; Z)) \cdot\left(\nabla_{z} f^{\varepsilon, \tau}\right)(t, \mathcal{Z}(t / \varepsilon ; Z))
$$

and

$$
\begin{aligned}
a\left(t, \mathcal{Z}\left(\frac{t}{\varepsilon} ; Z\right)\right) \cdot\left(\nabla_{z} f^{\varepsilon, \tau}\right)\left(t, \mathcal{Z}\left(\frac{t}{\varepsilon} ; Z\right)\right) & =a\left(t, \mathcal{Z}\left(\frac{t}{\varepsilon} ; Z\right)\right) \cdot{ }^{t} \partial \mathcal{Z}\left(-\frac{t}{\varepsilon} ; \mathcal{Z}\left(\frac{t}{\varepsilon} ; Z\right)\right)\left(\nabla_{Z} F^{\varepsilon, \tau}\right)(t, Z) \\
& =\partial \mathcal{Z}\left(-\frac{t}{\varepsilon} ; \mathcal{Z}\left(\frac{t}{\varepsilon} ; Z\right)\right) a\left(t, \mathcal{Z}\left(\frac{t}{\varepsilon} ; Z\right)\right) \cdot \nabla_{Z} F^{\varepsilon, \tau}(t, Z) .
\end{aligned}
$$

With respect to the new unknowns, the problem (2), (3) becomes

$$
\begin{aligned}
& \partial_{t} F^{\varepsilon, \tau}+\partial \mathcal{Z}\left(-\frac{t}{\varepsilon} ; \mathcal{Z}\left(\frac{t}{\varepsilon} ; Z\right)\right) a\left(t, \mathcal{Z}\left(\frac{t}{\varepsilon} ; Z\right)\right) \cdot \nabla_{Z} F^{\varepsilon, \tau}(t, Z) \\
&=\frac{\tilde{Q}_{F P L}}{\tau}\left(F^{\varepsilon, \tau}\left(t, \mathcal{Z}\left(-\frac{t}{\varepsilon} ; \cdot\right)\right)\right)\left(\mathcal{Z}\left(\frac{t}{\varepsilon} ; Z\right)\right) \\
& F^{\varepsilon, \tau}(0, X, V)=f^{\text {in }}(X, V), \quad Z=(X, V) \in \mathbb{R}^{3} \times \mathbb{R}^{3} .
\end{aligned}
$$

We introduce the family $(\varphi(s))_{s \in \mathbb{R}}$ given by

$$
\varphi(s) a=\partial \mathcal{Z}(-s ; \mathcal{Z}(s ; \cdot)) a(\mathcal{Z}(s ; \cdot))
$$

for any vector field $a=a(t, z)$, and also the notation $G_{s}=G \circ \mathcal{Z}(s ; \cdot)$ for any presence density $G$. Therefore the equation (4) writes

$$
\partial_{t} F^{\varepsilon, \tau}+\varphi(t / \varepsilon)(a(t)) \cdot \nabla_{X, V} F^{\varepsilon, \tau}=\frac{\tilde{Q}_{F P L}}{\tau}\left(F^{\varepsilon, \tau}(t)_{-t / \varepsilon}\right)_{t / \varepsilon}, \quad(t, X, V) \in \mathbb{R}_{+} \times \mathbb{R}^{3} \times \mathbb{R}^{3} .
$$

Notice that the transport term $\varphi(t / \varepsilon)(a(t)) \cdot \nabla_{X, V} F^{\varepsilon, \tau}(t)$ has three dependencies with respect to time : 
- the dependence $t \rightarrow F^{\varepsilon, \tau}(t)$ is slow, since the new presence density $F^{\varepsilon, \tau}(t)$ has been obtained from the old presence density $f^{\varepsilon, \tau}(t)$, after smoothing out the fast oscillations due to the cyclotronic motion ;

- the dependence $t \rightarrow a(t)$ is slow, since the parallel dynamics evolves on a much larger time scale than the perpendicular dynamics ;

- the dependence $t \rightarrow \varphi(s=t / \varepsilon)$ is fast, corresponding to the time scale given by the cyclotronic period.

Similarly, the collision term evolves on both slow and fast time scales, the dependence $t \rightarrow F^{\varepsilon, \tau}(t)$ being slow, and the translations along the flow of $\frac{b}{\varepsilon} \cdot \nabla_{x, v}$ being fast. We are faced to a two time scale problem, and thus, the limit model when $\varepsilon \searrow 0$ comes by averaging with respect to the fast time variable, while keeping frozen the slow time variable

$$
\partial_{t} F^{\tau}+\langle a(t)\rangle \cdot \nabla_{X, V} F^{\tau}=\frac{1}{\tau}\left\langle\tilde{Q}_{F P L}\right\rangle\left(F^{\tau}(t)\right), \quad(t, X, V) \in \mathbb{R}_{+} \times \mathbb{R}^{3} \times \mathbb{R}^{3}
$$

where

$$
\langle a\rangle=\frac{1}{T_{c}} \int_{0}^{T_{c}} \varphi(s) a \mathrm{~d} s,\left\langle\tilde{Q}_{F P L}\right\rangle(F)=\frac{1}{T_{c}} \int_{0}^{T_{c}} \tilde{Q}_{F P L}\left(F_{-s}\right)_{s} \mathrm{~d} s .
$$

After performing the homogenization with respect to the cyclotronic motion, we focus on the second asymptotic procedure, that is, $\tau=T_{\text {coll }} / T_{\|} \rightarrow 0$. We deduce that at any time $t$, the limit presence density $F(t)=\lim _{\tau \searrow 0} F^{\tau}(t)$ is an equilibrium

$$
\left\langle\tilde{Q}_{F P L}\right\rangle(F(t))=0, \quad t>0 .
$$

As in the case of the standard Fokker-Planck-Landau kernel, we prove that any equilibrium is parametrized by a set of moments (see Proposition 4.5), whose evolutions are obtained thanks to the conservation laws associated to the collision invariants (see Proposition 4.4). We mention that, due to the gyro-average effect in the plane orthogonal to the magnetic lines, the fluid model is a system of conservation laws in the parallel direction. This model does not depend on the specific form of the collision kernel, but only on its equilibria, invariants and production entropy. Therefore, we formulate our main result in terms of general collision kernels verifying the $H$ Theorem satisfied by the Fokker-Planck-Landau operator cf. Theorem 4.1. For any $T>0$, the notation $\mathcal{M}_{T}$ stands for the Maxwellian of temperature $T$ i.e., $\mathcal{M}_{T}(w)=(2 \pi T)^{-N / 2} \exp \left(-|w|^{2} / 2 T\right), w \in \mathbb{R}^{N}$.

Theorem 1.1 Let $Q$ be a local in space operator, homogeneous of degree d, verifying the statements 1. and 2. of Theorem 4.1, and whose equilibria are the Maxwellians. We denote by $\left(f^{\varepsilon, \tau}\right)_{\varepsilon, \tau>0}$ the solutions of the problems (2), (3) (with uniform electromagnetic field and collision operator $Q$ ), where $\varepsilon, \tau$ are small parameters characterizing the regime (1). Therefore we have

$$
\lim _{\tau \searrow 0 \varepsilon \searrow 0} \lim _{\varepsilon}^{\varepsilon, \tau}(t, \mathcal{X}(t ; X, V), \mathcal{V}(t ; X, V))=F(t, X, V)
$$

where

$$
F(t, X, V)=\omega^{2} n \mathcal{M}_{\frac{\mu \theta}{\mu-\theta}}(\bar{V}-\bar{U}) \mathcal{M}_{\theta}\left(V_{3}-U_{3}\right) \mathcal{M}_{\mu}\left(\omega \bar{X}+{ }^{\perp} \bar{V}-\bar{Y}\right)
$$


and $n\left(t, X_{3}\right), \theta\left(t, X_{3}\right), \mu\left(t, X_{3}\right), U\left(t, X_{3}\right), \bar{Y}\left(t, X_{3}\right)$ verify the equations

$$
\partial_{t} n+\partial_{X_{3}}\left(n U_{3}\right)=0, \quad\left(t, X_{3}\right) \in \mathbb{R}_{+} \times \mathbb{R}
$$

$$
\begin{gathered}
\partial_{t}(n U)+\partial_{X_{3}}\left(n U_{3} U+n^{t}(0,0, \theta)\right)=n \frac{q}{m}{ }^{t}\left(0,0, E_{3}(t)\right), \quad\left(t, X_{3}\right) \in \mathbb{R}_{+} \times \mathbb{R} \\
\partial_{t}(n \bar{Y})+\partial_{X_{3}}\left(n U_{3} \bar{Y}\right)=n \frac{q}{m}{ }^{\perp} \bar{E}(t), \quad\left(t, X_{3}\right) \in \mathbb{R}_{+} \times \mathbb{R}
\end{gathered}
$$

$\partial_{t}\left[n\left(\frac{\mu \theta}{\mu-\theta}+\frac{\theta}{2}+\frac{|U|^{2}}{2}\right)\right]+\partial_{X_{3}}\left[n U_{3}\left(\frac{\mu \theta}{\mu-\theta}+\frac{3 \theta}{2}+\frac{|U|^{2}}{2}\right)\right]=n U_{3} \frac{q}{m} E_{3}(t)$

$\partial_{t}\left[n\left(\mu+\frac{|\bar{Y}|^{2}}{2}-\frac{\mu \theta}{\mu-\theta}-\frac{|\bar{U}|^{2}}{2}\right)\right]+\partial_{X_{3}}\left[n U_{3}\left(\mu+\frac{|\bar{Y}|^{2}}{2}-\frac{\mu \theta}{\mu-\theta}-\frac{|\bar{U}|^{2}}{2}\right)\right]=n \frac{q}{m} \bar{Y} \cdot{ }^{\perp} \bar{E}$

and the inequality

$$
\partial_{t}\left(n \ln \frac{n}{\frac{\mu^{2} \theta^{3 / 2}}{\mu-\theta}}\right)+\partial_{X_{3}}\left(n U_{3} \ln \frac{n}{\frac{\mu^{2} \theta^{3 / 2}}{\mu-\theta}}\right) \leq 0, \quad\left(t, X_{3}\right) \in \mathbb{R}_{+} \times \mathbb{R} .
$$

The initial conditions are given by

$$
\begin{gathered}
\int_{\mathbb{R}^{2}} \int_{\mathbb{R}^{3}} f^{\text {in }} \mathrm{d} V \mathrm{~d} \bar{X}=n^{\text {in }}, \int_{\mathbb{R}^{2}} \int_{\mathbb{R}^{3}} f^{\text {in }} V \mathrm{~d} V \mathrm{~d} \bar{X}=n^{\text {in }} U^{\text {in }}, \int_{\mathbb{R}^{2}} \int_{\mathbb{R}^{3}} f^{\text {in }}\left(\omega \bar{X}+{ }^{\perp} \bar{V}\right) \mathrm{d} V \mathrm{~d} \bar{X}=n^{\text {in }} \bar{Y}^{\text {in }} \\
\int_{\mathbb{R}^{2}} \int_{\mathbb{R}^{3}} f^{\text {in }} \frac{\left|V-U^{\text {in }}\right|^{2}}{2} \mathrm{~d} V \mathrm{~d} \bar{X}=n^{\text {in }}\left(\frac{\mu^{\text {in }} \theta^{\text {in }}}{\mu^{\text {in }}-\theta^{\text {in }}}+\frac{\theta^{\text {in }}}{2}\right) \\
\int_{\mathbb{R}^{2}} \int_{\mathbb{R}^{3}} f^{\text {in }} \frac{\left|\omega \bar{X}+{ }^{\perp} \bar{V}-\bar{Y}^{\text {in }}\right|^{2}-|\bar{V}|^{2}}{2} \mathrm{~d} V \mathrm{~d} \bar{X}=n^{\text {in }}\left(\mu^{\text {in }}-\frac{\mu^{\text {in }} \theta^{\text {in }}}{\mu^{\text {in }}-\theta^{\text {in }}}\right) .
\end{gathered}
$$

Our paper is organized as follows. The properties of the Fokker-Planck-Landau kernel are recalled in Section 2. The moment method, for the derivation of the Euler equations, is revisited in Section 3. In Section 4 we concentrate on the average collision operator. We investigate the structure of its equilibria and invariants, in order to apply the moment method, in the gyro-kinetic framework. The last section is devoted to the derivation of the Euler equations, corresponding to the average collision mechanism.

\section{The Fokker-Planck-Landau kernel}

We recall briefly the main properties of the Fokker-Planck-Landau collision kernel $[17,18,19,20]$. 
Proposition 2.1 (Mass, momentum, kinetic energy)

For any smooth distribution function $f=f(v)$, with nice decay at infinity (for example compactly supported), we have

$$
\int_{\mathbb{R}^{3}} Q_{F P L}(f) \mathrm{d} v=0, \quad \int_{\mathbb{R}^{3}} Q_{F P L}(f) v \mathrm{~d} v=0, \quad \int_{\mathbb{R}^{3}} Q_{F P L}(f) \frac{|v|^{2}}{2} \mathrm{~d} v=0 .
$$

Proof. We introduce the notation

$A\left(v, v^{\prime}\right)=\sigma\left(\left|v-v^{\prime}\right|\right)\left|v-v^{\prime}\right|^{3} S\left(v-v^{\prime}\right)\left[f\left(v^{\prime}\right)(\nabla f)(v)-f(v)(\nabla f)\left(v^{\prime}\right)\right], \quad v, v^{\prime} \in \mathbb{R}^{3}, v \neq v^{\prime}$ such that

$$
Q_{F P L}(f)(v)=\operatorname{div}_{v} \int_{\mathbb{R}^{3}} A\left(v, v^{\prime}\right) \mathrm{d} v^{\prime}, \quad v \in \mathbb{R}^{3} .
$$

Obviously we have

$$
\int_{\mathbb{R}^{3}} Q_{F P L}(f) \mathrm{d} v=\int_{\mathbb{R}^{3}} \operatorname{div}_{v} \int_{\mathbb{R}^{3}} A\left(v, v^{\prime}\right) \mathrm{d} v^{\prime} \mathrm{d} v=0 .
$$

The momentum balance follows immediately by integration by parts and thanks to the anti-symmetry of $A$

$$
A\left(v, v^{\prime}\right)+A\left(v^{\prime}, v\right)=0, \quad v, v^{\prime} \in \mathbb{R}^{3}, \quad v \neq v^{\prime} .
$$

We obtain

$$
\int_{\mathbb{R}^{3}} Q_{F P L}(f) v \mathrm{~d} v=-\int_{\mathbb{R}^{3}} \int_{\mathbb{R}^{3}} A\left(v, v^{\prime}\right) \mathrm{d} v^{\prime} \mathrm{d} v=-\frac{1}{2} \int_{\mathbb{R}^{3} \times \mathbb{R}^{3}}[\underbrace{A\left(v, v^{\prime}\right)+A\left(v^{\prime}, v\right)}_{=0}] \mathrm{d}\left(v, v^{\prime}\right)=0 .
$$

Similarly, taking into account that ${ }^{t} S\left(v-v^{\prime}\right)\left(v-v^{\prime}\right)=S\left(v-v^{\prime}\right)\left(v-v^{\prime}\right)=0$, one gets

$$
\begin{aligned}
\int_{\mathbb{R}^{3}} Q_{F P L}(f) \frac{|v|^{2}}{2} \mathrm{~d} v & =-\int_{\mathbb{R}^{3}} \int_{\mathbb{R}^{3}} A\left(v, v^{\prime}\right) \mathrm{d} v^{\prime} \cdot v \mathrm{~d} v \\
& =-\int_{\mathbb{R}^{3}} \int_{\mathbb{R}^{3}} A\left(v^{\prime}, v\right) \mathrm{d} v \cdot v^{\prime} \mathrm{d} v^{\prime} \\
& =-\frac{1}{2} \int_{\mathbb{R}^{3} \times \mathbb{R}^{3}}\left[\left(v, v^{\prime}\right) \cdot v+A\left(v^{\prime}, v\right) \cdot v^{\prime}\right] \mathrm{d}\left(v, v^{\prime}\right) \\
& =-\frac{1}{2} \int_{\mathbb{R}^{3} \times \mathbb{R}^{3}}[\underbrace{A\left(v, v^{\prime}\right)+A\left(v^{\prime}, v\right)}_{=0}] \cdot v^{\prime} \mathrm{d}\left(v, v^{\prime}\right)=0 .
\end{aligned}
$$

We will use the notion of collision equilibrium and collision invariant.

\section{Definition 2.1}

1. A distribution function $f=f(v)$ is called a collision equilibrium of the FokkerPlanck-Landau kernel iff $Q_{F P L}(f)=0$.

2. A function $c=c(v)$ is called a collision invariant of the Fokker-Planck-Landau kernel iff

$$
\int_{\mathbb{R}^{3}} Q_{F P L}(f)(v) c(v) \mathrm{d} v=0
$$

for any distribution function $f=f(v)$. 
We recall now a well known result, the $H$ Theorem, which allows us to identify the equilibria and invariants of the Fokker-Planck-Landau kernel.

Theorem 2.1 (H Theorem)

1. For any smooth distribution function $f=f(v)>0$, with nice decay at infinity, we have the inequality

$$
\int_{\mathbb{R}^{3}} \ln f(v) Q_{F P L}(f) \mathrm{d} v \leq 0
$$

2. Let $f=f(v)>0$ be a smooth distribution function, with nice decay at infinity. The following assertions are equivalent

(a) The distribution $f$ is an equilibrium, that is, $Q_{F P L}(f)=0$.

(b) The distribution $f$ achieves the equality in the inequality (8)

$$
\int_{\mathbb{R}^{3}} \ln f(v) Q_{F P L}(f) \mathrm{d} v=0 .
$$

(c) The function $c(v)=\ln f(v)$ is an invariant, that is

$$
\int_{\mathbb{R}^{3}} Q_{F P L}(g) c(v) \mathrm{d} v=0
$$

for any distribution function $g$.

\section{Proof.}

1. We use one more time integration by parts and Fubini Theorem, combined to the positivity of the matrices $S\left(v-v^{\prime}\right), v, v^{\prime} \in \mathbb{R}^{3}, v \neq v^{\prime}$

$$
S\left(v-v^{\prime}\right): \xi \otimes \xi=\frac{\left|\xi \wedge\left(v-v^{\prime}\right)\right|^{2}}{\left|v-v^{\prime}\right|^{2}}, \quad v \neq v^{\prime}, \quad \xi \in \mathbb{R}^{3}
$$

It is easily seen that

$$
\begin{aligned}
\int_{\mathbb{R}^{3}} \ln f(v) Q_{F P L}(f) \mathrm{d} v & =-\frac{1}{2} \int_{\mathbb{R}^{3} \times \mathbb{R}^{3}}\left[(\nabla \ln f)(v)-(\nabla \ln f)\left(v^{\prime}\right)\right] \cdot A\left(v, v^{\prime}\right) \mathrm{d}\left(v, v^{\prime}\right) \\
& =-\frac{1}{2} \int_{\mathbb{R}^{3} \times \mathbb{R}^{3}} \sigma\left(\left|v-v^{\prime}\right|\right)\left|v-v^{\prime}\right|^{3} f(v) f\left(v^{\prime}\right) S\left(v-v^{\prime}\right) \\
& :\left[(\nabla \ln f)(v)-(\nabla \ln f)\left(v^{\prime}\right)\right] \otimes\left[(\nabla \ln f)(v)-(\nabla \ln f)\left(v^{\prime}\right)\right] \mathrm{d}\left(v, v^{\prime}\right) \\
& =-\frac{1}{2} \int_{\mathbb{R}^{3} \times \mathbb{R}^{3}} \sigma\left(\left|v-v^{\prime}\right|\right)\left|v-v^{\prime}\right| f(v) f\left(v^{\prime}\right) \\
& \left|\left(v-v^{\prime}\right) \wedge\left[(\nabla \ln f)(v)-(\nabla \ln f)\left(v^{\prime}\right)\right]\right|^{2} \mathrm{~d}\left(v, v^{\prime}\right) \leq 0 .
\end{aligned}
$$

2. All the implications are straightforward, except for $(b) \Longrightarrow(a)$. In order to prove (a) when assuming $(b)$, observe that by the previous computations we have

$$
\int_{\mathbb{R}^{3} \times \mathbb{R}^{3}} \sigma\left(\left|v-v^{\prime}\right|\right)\left|v-v^{\prime}\right| f(v) f\left(v^{\prime}\right)\left|\left(v-v^{\prime}\right) \wedge\left[(\nabla \ln f)(v)-(\nabla \ln f)\left(v^{\prime}\right)\right]\right|^{2} \mathrm{~d}\left(v, v^{\prime}\right)=0
$$


and therefore

$$
\left(v-v^{\prime}\right) \wedge\left[(\nabla \ln f)(v)-(\nabla \ln f)\left(v^{\prime}\right)\right]=0, \quad v, v^{\prime} \in \mathbb{R}^{3}
$$

Taking $v^{\prime}=v+s \xi$, with $\xi \in \mathbb{R}^{3}, s \in \mathbb{R}$, one gets

$$
\xi \wedge \frac{(\nabla \ln f)(v+s \xi)-(\nabla \ln f)(v)}{s}=0
$$

and letting $s \rightarrow 0$ yields

$$
\xi \wedge \partial^{2} \ln f \xi=0, \quad \xi \in \mathbb{R}^{3} .
$$

The equality (9) implies immediately that for any $v \in \mathbb{R}^{3}$, there is $\alpha=\alpha(v) \in \mathbb{R}$ such that

$$
\partial^{2} \ln f(v)=\alpha(v) I_{3} .
$$

It is easily seen that $\nabla_{v} \alpha=0$. Actually we have

$$
\partial_{v_{2}} \partial_{v_{1}} \ln f=\partial_{v_{3}} \partial_{v_{1}} \ln f=0, \quad v \in \mathbb{R}^{3}
$$

saying that $\partial_{v_{1}} \ln f$ depends only on $v_{1}$. Therefore $\nabla_{v_{2}, v_{3}} \alpha(v)=\nabla_{v_{2}, v_{3}} \partial_{v_{1}}\left(\partial_{v_{1}} \ln f\right)=$ ${ }^{t}(0,0)$. Similarly we show that $\nabla_{v_{3}, v_{1}} \alpha={ }^{t}(0,0)$. Finally the function $\ln f$ writes

$$
\ln f(v)=\alpha \frac{|v|^{2}}{2}+\beta \cdot v+\gamma, \quad v \in \mathbb{R}^{3}
$$

with $\alpha, \gamma \in \mathbb{R}, \beta \in \mathbb{R}^{3}$, and we check immediately that $Q_{F P L}(f)=0$.

\section{The Euler equations}

The study of the equilibria and invariants plays a crucial role when deriving macroscopic limits from microscopic models $[1,2,23,24]$. When the collision frequency is very large, the presence density goes toward an equilibrium profile in velocity, parametrized by several macroscopic quantities, as density, mean velocity, temperature. The evolution of these quantities follows thanks to the collision invariants. Any invariant leads to a conservation law in the physical space phase. Appealing to the moment method, we derive the Euler equations from the Boltzmann equation cf. $[1,2]$. We intend to apply the same method for studying the fluid models in the gyro-kinetic framework. Before doing that, we revisit the moment method in the context of the standard Euler equations. More exactly, let us consider the fluctuations of the presence density $f$, due to the free transport and collisions

$$
\begin{gathered}
\partial_{t} f+v \cdot \nabla_{x} f=\frac{1}{\tau} Q_{F P L}(f), \quad(t, x, v) \in \mathbb{R}_{+} \times \mathbb{R}^{3} \times \mathbb{R}^{3} \\
f(0, x, v)=f^{\text {in }}(x, v), \quad(x, v) \in \mathbb{R}^{3} \times \mathbb{R}^{3} .
\end{gathered}
$$

When the collision mechanism dominates the transport (i.e., $\tau \searrow 0$ ), at any time $t$ and any position $x$, the distribution function $f(t, x, \cdot)$ becomes a collision equilibrium. By Theorem 2.1, we deduce that for any $t \in \mathbb{R}_{+}, x \in \mathbb{R}^{3}$, the function $\ln f(t, x, \cdot)$ is a 
linear combination of $1, v,|v|^{2} / 2 \mathrm{cf}$. (10). We obtain that $f$ is a Maxwellian, i.e., there are $n=n(t, x) \geq 0, u=u(t, x) \in \mathbb{R}^{3}, \theta=\theta(t, x)>0$ such that

$$
f(t, x, v)=M_{n(t, x), u(t, x), \theta(t, x)}(v), \quad M_{n, u, \theta}(v)=\frac{n}{(2 \pi \theta)^{3 / 2}} \exp \left(-\frac{|v-u|^{2}}{2 \theta}\right), \quad v \in \mathbb{R}^{3} .
$$

The macroscopic quantities $n(t, x), u(t, x), \theta(t, x)$ satisfy a system of conservation laws which comes by appealing to the collision invariants $1, v,|v|^{2} / 2$

$$
\begin{gathered}
\partial_{t} \int_{\mathbb{R}^{3}} f \mathrm{~d} v+\operatorname{div}_{x} \int_{\mathbb{R}^{3}} f v \mathrm{~d} v=\int_{\mathbb{R}^{3}} Q_{F P L}(f) \mathrm{d} v=0 \\
\partial_{t} \int_{\mathbb{R}^{3}} f v \mathrm{~d} v+\operatorname{div}_{x} \int_{\mathbb{R}^{3}} f v \otimes v \mathrm{~d} v=\int_{\mathbb{R}^{3}} Q_{F P L}(f) v \mathrm{~d} v=0 \\
\partial_{t} \int_{\mathbb{R}^{3}} f \frac{|v|^{2}}{2} \mathrm{~d} v+\operatorname{div}_{x} \int_{\mathbb{R}^{3}} f \frac{|v|^{2}}{2} v \mathrm{~d} v=\int_{\mathbb{R}^{3}} Q_{F P L}(f) \frac{|v|^{2}}{2} \mathrm{~d} v=0 .
\end{gathered}
$$

It is enough to express the moments of $f$ in terms of the macroscopic quantities $n, u, \theta$. After straightforward computations, the system (12), (13), (14) leads to the Euler equations

$$
\begin{gathered}
\partial_{t} n+\operatorname{div}_{x}(n u)=0, \quad(t, x) \in \mathbb{R}_{+} \times \mathbb{R}^{3} \\
\partial_{t}(n u)+\operatorname{div}_{x}(n u \otimes u)+\nabla_{x}(n \theta)=0, \quad(t, x) \in \mathbb{R}_{+} \times \mathbb{R}^{3} \\
\partial_{t}\left[n\left(\frac{3}{2} \theta+\frac{|u|^{2}}{2}\right)\right]+\operatorname{div}_{x}\left[n\left(\frac{5}{2} \theta+\frac{|u|^{2}}{2}\right) u\right]=0, \quad(t, x) \in \mathbb{R}_{+} \times \mathbb{R}^{3} .
\end{gathered}
$$

The above system also possesses an entropy inequality. Multiplying (11) by $(1+\ln f)$ we obtain

$$
\partial_{t}(f \ln f)+v \cdot \nabla_{x}(f \ln f)=(1+\ln f) Q_{F P L}(f)
$$

and integrating in velocity, using the mass balance and the production entropy inequality (8) yield

$$
\partial_{t} \int_{\mathbb{R}^{3}} f \ln f \mathrm{~d} v+\operatorname{div}_{x} \int_{\mathbb{R}^{3}} f \ln f v \mathrm{~d} v=\int_{\mathbb{R}^{3}}(1+\ln f) Q_{F P L}(f) \mathrm{d} v \leq 0 .
$$

Finally, as $f=M_{n, u, \theta}$ we have

$$
\begin{gathered}
\int_{\mathbb{R}^{3}} f \ln f \mathrm{~d} v=\int_{\mathbb{R}^{3}} f\left[\ln \left(\frac{n}{(2 \pi \theta)^{3 / 2}}\right)-\frac{|v-u|^{2}}{2 \theta}\right] \mathrm{d} v=n \ln \left(\frac{n}{(2 \pi \theta)^{3 / 2}}\right)-\frac{3}{2} n \\
\int_{\mathbb{R}^{3}} f \ln f v \mathrm{~d} v=\int_{\mathbb{R}^{3}} f\left[\ln \left(\frac{n}{(2 \pi \theta)^{3 / 2}}\right)-\frac{|v-u|^{2}}{2 \theta}\right] v \mathrm{~d} v=n \ln \left(\frac{n}{(2 \pi \theta)^{3 / 2}}\right) u-\frac{3}{2} n u .
\end{gathered}
$$

Therefore we obtain

$$
\partial_{t}\left[n\left(\ln \frac{n}{(2 \pi \theta)^{3 / 2}}-\frac{3}{2}\right)\right]+\operatorname{div}_{x}\left[n\left(\ln \frac{n}{(2 \pi \theta)^{3 / 2}}-\frac{3}{2}\right) u\right] \leq 0
$$

or equivalently, thanks to the continuity equation (15)

$$
\partial_{t}\left[n \ln \left(\frac{n}{\theta^{3 / 2}}\right)\right]+\operatorname{div}_{x}\left[n \ln \left(\frac{n}{\theta^{3 / 2}}\right) u\right] \leq 0 .
$$


We remark that the limit model (15), (16), (17), (18) does not depend on the expression of the collision kernel (here the Fokker-Planck-Landau collision operator), but only on the properties mentionned in Theorem 2.1. It is well known that the Boltzmann operator also verifies the statement in Theorem 2.1. Thus if in (11) the Fokker-PlanckLandau kernel is replaced by the Boltzmann kernel, we obtain the same limit model (15), (16), (17), (18). Similarly, we will see that the fluid models with strong magnetic fields will depend only on the physical balances and entropy inequality, but not on the specific form of the effective collision kernel. In other words we do not need to compute explicitly the average collision kernel, but only to establish a corresponding $H$ theorem, in order to investigate the entropy production and the structure of its equilibria and invariants.

\section{The average collision operator}

A collision operator acts only in velocity : to any velocity distribution function $f=$ $f(v)$, it associates another velocity distribution function $Q(f)(v), v \in \mathbb{R}^{3}$. In particular, if $f=f(x, v)$ and $g=g(x, v)$ are two presence densities such that $f\left(x^{\star}, v\right)=$ $g\left(x^{\star}, v\right), v \in \mathbb{R}^{3}$, for some $x^{\star}$, then $Q\left(f\left(x^{\star}, \cdot\right)\right)(v)=Q\left(g\left(x^{\star}, \cdot\right)\right)(v), v \in \mathbb{R}^{3}$. We say that the operator $Q$ is local in space. It is also possible to consider the collision operator as a transformation on the set of presence densities : to any presence density $f=f(x, v)$, it associates another function of $(x, v)$, given by $\tilde{Q}(f)(x, v)=Q(f(x, \cdot))(v)$. From now on, the collision operators are supposed acting on the set of presence densities, but we use the notation $Q$ instead of $\tilde{Q}$. Notice that the space locality writes : for any presence densities $f=f(x, v), g=g(x, v)$ and any $x^{\star} \in \mathbb{R}^{3}$ such that $f\left(x^{\star}, v\right)=g\left(x^{\star}, v\right), v \in \mathbb{R}^{3}$, we have $Q(f)\left(x^{\star}, v\right)=Q(g)\left(x^{\star}, v\right), v \in \mathbb{R}^{3}$. The notions of collision equilibrium and invariant become

Definition 4.1 Let $Q$ be a collision operator acting on the phase space $(x, v) \in \mathbb{R}^{3} \times \mathbb{R}^{3}$.

1. A presence density $f=f(x, v)$ is called an equilibrium of $Q$ iff

$$
Q(f)(x, v)=0,(x, v) \in \mathbb{R}^{3} \times \mathbb{R}^{3} .
$$

2. A function $c=c(x, v)$ is called an invariant of $Q$ iff

$$
\int_{\mathbb{R}^{3}} \int_{\mathbb{R}^{3}} Q(f)(x, v) c(x, v) \mathrm{d} v \mathrm{~d} x=0
$$

for any presence density $f=f(x, v)$.

Theorem 2.1 adapts easily to presence densities on the phase space $(x, v) \in \mathbb{R}^{3} \times \mathbb{R}^{3}$.

Theorem 4.1 Let $Q$ be the operator defined by

$$
Q(f)(x, v)=Q_{F P L}(f(x, \cdot))(v), \quad(x, v) \in \mathbb{R}^{3} \times \mathbb{R}^{3}
$$

for any smooth presence density $f=f(x, v)$, with nice decay at infinity. 
1. For any presence density $f=f(x, v)>0$, we have the inequality

$$
\int_{\mathbb{R}^{3}} \int_{\mathbb{R}^{3}} \ln f(x, v) Q(f)(x, v) \mathrm{d} v \mathrm{~d} x \leq 0 .
$$

2. Let $f=f(x, v)>0$ be a presence density. The following assertions are equivalent

(a) The presence density $f$ is an equilibrium of $Q$, that is, $Q(f)=0$.

(b) The presence density $f$ achieves the equality in the inequality (19)

$$
\int_{\mathbb{R}^{3}} \int_{\mathbb{R}^{3}} \ln f(x, v) Q(f)(x, v) \mathrm{d} v \mathrm{~d} x=0 .
$$

(c) The function $c(x, v)=\ln f(x, v)$ is an invariant of $Q$, that is

$$
\int_{\mathbb{R}^{3}} \int_{\mathbb{R}^{3}} Q(g)(x, v) c(x, v) \mathrm{d} v \mathrm{~d} x=0
$$

for any presence density $g$.

\section{Proof.}

1. The inequality (19) is a direct consequence of the inequality (8)

$$
\int_{\mathbb{R}^{3}} \int_{\mathbb{R}^{3}} \ln f(x, v) Q(f)(x, v) \mathrm{d} v \mathrm{~d} x=\int_{\mathbb{R}^{3}}\left(\int_{\mathbb{R}^{3}} \ln f(x, v) Q_{F P L}(f(x, \cdot))(v) \mathrm{d} v\right) \mathrm{d} x \leq 0 .
$$

2. Clearly $(a)$ implies $(b)$. Conversely, let $f=f(x, v)$ verifying

$$
\int_{\mathbb{R}^{3}} \int_{\mathbb{R}^{3}} \ln f(x, v) Q(f)(x, v) \mathrm{d} v \mathrm{~d} x=0 .
$$

By (8) we know that for any $x \in \mathbb{R}^{3}$ we have

$$
\int_{\mathbb{R}^{3}} \ln f(x, v) Q(f)(x, v) \mathrm{d} v=\int_{\mathbb{R}^{3}} \ln f(x, v) Q_{F P L}(f(x, \cdot))(v) \mathrm{d} v \leq 0
$$

and by combining to $(20)$, we deduce that

$$
\int_{\mathbb{R}^{3}} \ln f(x, v) Q_{F P L}(f(x, \cdot))(v) \mathrm{d} v=0, \quad x \in \mathbb{R}^{3} .
$$

Appealing to the implication $(b) \Longrightarrow(a)$ of Theorem 2.1, one gets that $Q(f)(x, v)=$ $Q_{F P L}(f(x, \cdot))(v)=0,(x, v) \in \mathbb{R}^{3} \times \mathbb{R}^{3}$, saying that $f$ is an equilibrium of $Q$.

Let us check that $(b)$ implies $(c)$. Let $f=f(x, v)$ be a presence density verifying (20). Thanks to the previous arguments, we know that $f(x, \cdot)$ is an equilibrium of $Q_{F P L}$ for any $x \in \mathbb{R}^{3}$, and therefore a Maxwellian, parametrized by $n(x), u(x), \theta(x)$. Therefore, for any $x \in \mathbb{R}^{3}, c(x, v)=\ln f(x, v)$ is a linear combination of $1, v,|v|^{2} / 2$

$$
\ln f(x, v)=\ln M_{n(x), u(x), \theta(x)}(v)=\ln \frac{n(x)}{(2 \pi \theta(x))^{3 / 2}}-\frac{|v-u(x)|^{2}}{2 \theta(x)}
$$

and thus an invariant of $Q_{F P L}$ (cf. Proposition 2.1). We deduce that for any presence density $g=g(x, v)$ we have

$$
\int_{\mathbb{R}^{3}} \int_{\mathbb{R}^{3}} Q(g) c(x, v) \mathrm{d} v \mathrm{~d} x=\int_{\mathbb{R}^{3}}\left(\int_{\mathbb{R}^{3}} Q_{F P L}(g(x, \cdot))(v) c(x, v) \mathrm{d} v\right) \mathrm{d} x=0
$$

saying that $c=\ln f$ is an invariant of $Q$.

For checking $(c) \Longrightarrow(b)$, we take $g=e^{c}=f$. 


\section{Remark 4.1}

1. The previous arguments show that the presence density $f=f(x, v)$ is an equilibrium of the Fokker-Planck-Landau operator iff for any $x \in \mathbb{R}^{3}$ there are $n(x), u(x), \theta(x)$ such that $f(x, \cdot)=M_{n(x), u(x), \theta(x)}$.

2. A function $c=c(x, v)$ is an invariant of the Fokker-Planck-Landau operator iff for any $x \in \mathbb{R}^{3}, c(x, \cdot)$ is a linear combination (with coefficients depending on $x$ ) of $1, v,|v|^{2} / 2$.

From now on, we work with local in space operators $Q$ verifying the statements in Theorem 4.1, whose equilibria are the Maxwellian $M_{n(x), u(x), \theta(x)}(v), n \geq 0, \theta>0$. We have noticed that the derivation of the fluid limit, in the gyro-kinetic framework relies essentially on the identification of the equilibria and invariants of the average collision kernel. We will concentrate on how the properties of a collision operator propagate to its average version. For example, if $Q$ is local in space, then $\langle Q\rangle$ is local with respect to the parallel space coordinate. This is a consequence of the fact that $x_{3}$ is the only space coordinate which is left invariant by the flow $\mathcal{Z}=(\mathcal{X}, \mathcal{V})$. It is not the case of the perpendicular space coordinates. Actually only the Larmor center is left invariant by this flow

$$
\overline{\mathcal{X}}(t ; x, v)+\frac{{ }^{\perp} \overline{\mathcal{V}}(t ; x, v)}{\omega}=\bar{x}+\frac{{ }^{\perp} \bar{v}}{\omega} .
$$

Proposition 4.1 Let $Q$ be a local in space operator. Then the average operator

$$
\langle Q\rangle(F)=\frac{1}{T_{c}} \int_{0}^{T_{c}} Q\left(F_{-t}\right)_{t} \mathrm{~d} t
$$

is local with respect to $X_{3}$.

Proof. Let $F=F(X, V), G=G(X, V)$ be two presence densities such that for some $X_{3}^{\star} \in \mathbb{R}$ we have

$$
F\left(\bar{X}, X_{3}^{\star}, V\right)=G\left(\bar{X}, X_{3}^{\star}, V\right), \quad(\bar{X}, V) \in \mathbb{R}^{5} .
$$

As $\mathcal{X}_{3}\left(-t ; \bar{x}, X_{3}^{\star}, v\right)=X_{3}^{\star}$, we have for any $t \in \mathbb{R},(\bar{x}, v) \in \mathbb{R}^{5}$

$$
F_{-t}\left(\bar{x}, X_{3}^{\star}, v\right)=F\left(\mathcal{Z}\left(-t ; \bar{x}, X_{3}^{\star}, v\right)\right)=G\left(\mathcal{Z}\left(-t ; \bar{x}, X_{3}^{\star}, v\right)\right)=G_{-t}\left(\bar{x}, X_{3}^{\star}, v\right) .
$$

In particular

$$
F_{-t}\left(\bar{x}, X_{3}^{\star}, \cdot\right)=G_{-t}\left(\bar{x}, X_{3}^{\star}, \cdot\right), \quad t \in \mathbb{R}, \quad \bar{x} \in \mathbb{R}^{2}
$$

and since $Q$ is local in space, we deduce that

$$
Q\left(F_{-t}\right)\left(\bar{x}, X_{3}^{\star}, \cdot\right)=Q\left(G_{-t}\right)\left(\bar{x}, X_{3}^{\star}, \cdot\right), \quad t \in \mathbb{R}, \quad \bar{x} \in \mathbb{R}^{2}
$$

saying that

$$
Q\left(F_{-t}\right)\left(\cdot, \cdot, X_{3}^{\star}, \cdot\right)=Q\left(G_{-t}\right)\left(\cdot, \cdot, X_{3}^{\star}, \cdot\right), \quad t \in \mathbb{R} .
$$

Using one more time the invariance of the parallel space coordinate along the flow $\mathcal{Z}=(\mathcal{X}, \mathcal{V})$, we obtain

$$
\begin{aligned}
\langle Q\rangle(F)\left(\bar{X}, X_{3}^{\star}, V\right) & =\frac{1}{T_{c}} \int_{0}^{T_{c}} Q\left(F_{-t}\right)\left(\mathcal{Z}\left(t ; \bar{X}, X_{3}^{\star}, V\right)\right) \mathrm{d} t \\
& =\frac{1}{T_{c}} \int_{0}^{T_{c}} Q\left(G_{-t}\right)\left(\mathcal{Z}\left(t ; \bar{X}, X_{3}^{\star}, V\right)\right) \mathrm{d} t \\
& =\langle Q\rangle(G)\left(\bar{X}, X_{3}^{\star}, V\right) .
\end{aligned}
$$


The properties mentionned in Theorem 4.1 allow us to characterize the equilibria and invariants of the average collision kernel.

Proposition 4.2 Let $Q$ be an operator verifying the statements 1. and 2. of Theorem 4.1.

1. A presence density $F=F(X, V)$ is an equilibrium of $\langle Q\rangle$ iff for any $t \in \mathbb{R}$ the presence density $F_{-t}$ is an equilibrium of $Q$.

2. A function $C=C(X, V)$ is an invariant of $\langle Q\rangle$ iff for any $t \in \mathbb{R}$, the function $C_{-t}$ is an invariant of $Q$.

Proof.

1. Obviously, if $F$ is such that $Q\left(F_{-t}\right)=0, t \in \mathbb{R}$, then

$$
\langle Q\rangle(F)=\frac{1}{T_{c}} \int_{0}^{T_{c}} Q\left(F_{-t}\right)_{t} \mathrm{~d} t=0 .
$$

Conversely, let $F=F(X, V)$ be an equilibrium of $\langle Q\rangle$. We deduce that

$$
\begin{aligned}
0 & =\int_{\mathbb{R}^{3}} \int_{\mathbb{R}^{3}}\langle Q\rangle(F) \ln F(X, V) \mathrm{d} V \mathrm{~d} X=\int_{\mathbb{R}^{3}} \int_{\mathbb{R}^{3}} \frac{1}{T_{c}} \int_{0}^{T_{c}} Q\left(F_{-t}\right)_{t} \mathrm{~d} t \ln F \mathrm{~d} V \mathrm{~d} X \\
& =\frac{1}{T_{c}} \int_{0}^{T_{c}} \int_{\mathbb{R}^{3}} \int_{\mathbb{R}^{3}} Q\left(F_{-t}\right)_{t} \ln F \mathrm{~d} V \mathrm{~d} X \mathrm{~d} t \\
& =\frac{1}{T_{c}} \int_{0}^{T_{c}} \int_{\mathbb{R}^{3}} \int_{\mathbb{R}^{3}} Q\left(F_{-t}\right) \ln F_{-t} \mathrm{~d} v \mathrm{~d} x \mathrm{~d} t .
\end{aligned}
$$

Notice that for any $t \in \mathbb{R}$ we have cf. (19)

$$
\int_{\mathbb{R}^{3}} \int_{\mathbb{R}^{3}} Q\left(F_{-t}\right) \ln F_{-t} \mathrm{~d} v \mathrm{~d} x \leq 0
$$

and therefore

$$
\int_{\mathbb{R}^{3}} \int_{\mathbb{R}^{3}} Q\left(F_{-t}\right) \ln F_{-t} \mathrm{~d} v \mathrm{~d} x=0, \quad t \in \mathbb{R} .
$$

We deduce that $Q\left(F_{-t}\right)=0$ for any $t \in \mathbb{R}$ (we use the implication $(b) \Longrightarrow(a)$ in the second statement of Theorem 4.1).

2. Let $C=C(X, V)$ be a function such that $C_{-t}$ is an invariant of $Q$ for any $t \in \mathbb{R}$. Then for any presence density $G=G(X, V)$ we have

$$
\begin{aligned}
\int_{\mathbb{R}^{3}} \int_{\mathbb{R}^{3}}\langle Q\rangle(G) C(X, V) \mathrm{d} V \mathrm{~d} X & =\int_{\mathbb{R}^{3}} \int_{\mathbb{R}^{3}} \frac{1}{T_{c}} \int_{0}^{T_{c}} Q\left(G_{-t}\right)_{t} \mathrm{~d} t C(X, V) \mathrm{d} V \mathrm{~d} X \\
& =\frac{1}{T_{c}} \int_{0}^{T_{c}} \int_{\mathbb{R}^{3}} \int_{\mathbb{R}^{3}} Q\left(G_{-t}\right)_{t} C(X, V) \mathrm{d} V \mathrm{~d} X \mathrm{~d} t \\
& =\frac{1}{T_{c}} \int_{0}^{T_{c}} \underbrace{\int_{\mathbb{R}^{3}} \int_{\mathbb{R}^{3}} Q\left(G_{-t}\right) C_{-t} \mathrm{~d} v \mathrm{~d} x}_{=0} \mathrm{~d} t=0 .
\end{aligned}
$$


Conversely, let $C=C(X, V)$ be an invariant of $\langle Q\rangle$, that is

$$
\int_{\mathbb{R}^{3}} \int_{\mathbb{R}^{3}}\langle Q\rangle(G) C(X, V) \mathrm{d} V \mathrm{~d} X=0
$$

for any presence density $G=G(X, V)$. Taking $G=e^{C}$ we obtain

$$
\begin{aligned}
0 & =\int_{\mathbb{R}^{3}} \int_{\mathbb{R}^{3}}\langle Q\rangle\left(e^{C}\right) C(X, V) \mathrm{d} V \mathrm{~d} X \\
& =\int_{\mathbb{R}^{3}} \int_{\mathbb{R}^{3}} \frac{1}{T_{c}} \int_{0}^{T_{c}} Q\left(\left(e^{C}\right)_{-t}\right)_{t} \mathrm{~d} t C(X, V) \mathrm{d} V \mathrm{~d} X \\
& =\frac{1}{T_{c}} \int_{0}^{T_{c}} \int_{\mathbb{R}^{3}} \int_{\mathbb{R}^{3}} Q\left(e^{C_{-t}}\right)_{t} C(X, V) \mathrm{d} V \mathrm{~d} X \mathrm{~d} t \\
& =\frac{1}{T_{c}} \int_{0}^{T_{c}} \int_{\mathbb{R}^{3}} \int_{\mathbb{R}^{3}} Q\left(e^{C_{-t}}\right) C_{-t} \mathrm{~d} v \mathrm{~d} x \mathrm{~d} t .
\end{aligned}
$$

As before, combining to the inequalities

$$
\int_{\mathbb{R}^{3}} \int_{\mathbb{R}^{3}} Q\left(e^{C_{-t}}\right) C_{-t} \mathrm{~d} v \mathrm{~d} x \leq 0, \quad t \in \mathbb{R}
$$

we deduce that

$$
\int_{\mathbb{R}^{3}} \int_{\mathbb{R}^{3}} Q\left(e^{C_{-t}}\right) C_{-t} \mathrm{~d} v \mathrm{~d} x=0, \quad t \in \mathbb{R}
$$

saying that $C_{-t}=\ln e^{C_{-t}}$ is an invariant of $Q$ for any $t \in \mathbb{R}$ (we have used the implication $(b) \Longrightarrow(c)$ in the second statement of Theorem 4.1).

\section{Remark 4.2}

As for the operator $Q$, a presence density $F=F(X, V)$ is an equilibrium of $\langle Q\rangle$ iff $C=\ln F$ is an invariant of $\langle Q\rangle$. Indeed, a presence density $F$ is an equilibrium of $\langle Q\rangle$ $\Longleftrightarrow F_{-t}$ is an equilibrium of $Q$ for any $t \in \mathbb{R} \Longleftrightarrow \ln F_{-t}$ is an invariant of $Q$ for any $t \in \mathbb{R} \Longleftrightarrow \ln F$ is an invariant of $\langle Q\rangle$.

In order to identify the invariants of $\langle Q\rangle$, we need the following lemma.

Lemma 4.1 Let $Q$ be an operator verifying the statements 1. and 2. of Theorem 4.1. Let $C=C(X, V)$ be an invariant of $\langle Q\rangle$. Then $b \cdot \nabla_{Z} C$ is also an invariant of $\langle Q\rangle$. Moreover $b \cdot \nabla_{Z}\left(b \cdot \nabla_{Z} C\right), b \cdot \nabla_{Z}\left(b \cdot \nabla_{Z}\left(b \cdot \nabla_{Z} C\right)\right), \ldots$ are invariants of $\langle Q\rangle$.

Proof. We know that $C_{t}$ is an invariant of $Q$ for any $t \in \mathbb{R}$. By linearity, we deduce that $\left(C_{t+h}-C_{t}\right) / h$ is an invariant of $Q$ for any $t \in \mathbb{R}, h \neq 0$. Passing to the limit when $h \rightarrow 0$, one gets that $\left(b \cdot \nabla_{Z} C\right)_{t}$ is an invariant of $Q$ for any $t \in \mathbb{R}$. By the second statement of Proposition 4.2, it follows that $b \cdot \nabla_{Z} C$ is an invariant of $\langle Q\rangle$.

We notice the following properties of the average kernel.

Proposition 4.3 Let $Q$ be an operator verifying the statements 1. and 2. of Theorem 4.1, and whose equilibria are the Maxwellians.

1. Let $\alpha=\alpha\left(X_{3}\right)$ be a function. If $C=C(X, V)$ is an invariant of $\langle Q\rangle$, then $\alpha C$ is also an invariant of $\langle Q\rangle$. 
2. Let $C=C(X, V)$ be an invariant of $\langle Q\rangle$. Then for any presence density $G=$ $G(X, V)$, we have

$$
\int_{\mathbb{R}^{2}} \int_{\mathbb{R}^{3}}\langle Q\rangle(G) C(X, V) \mathrm{d} V \mathrm{~d} \bar{X}=0, \quad X_{3} \in \mathbb{R}
$$

3. Assume also that the operator $Q$ is local in space, and homogeneous of degree $d$ $\left(Q(\lambda f)=\lambda^{d} Q(f)\right.$ for any presence density $f=f(x, v)$ and any $\left.\lambda \in \mathbb{R}_{+}\right)$. Then for any presence density $F=F(X, V)$ and function $\alpha=\alpha\left(X_{3}\right) \geq 0$, we have $\langle Q\rangle(\alpha F)=\alpha^{d}\langle Q\rangle(F)$ and

$$
\int_{\mathbb{R}^{2}} \int_{\mathbb{R}^{3}}\langle Q\rangle(F) \ln F \mathrm{~d} V \mathrm{~d} \bar{X} \leq 0, \quad X_{3} \in \mathbb{R} .
$$

\section{Proof.}

1. Let $C=C(X, V)$ be an invariant of $\langle Q\rangle$. By Proposition 4.2 we know that $C_{-t}$ is an invariant of $Q$, for any $t \in \mathbb{R}$. For any function $\alpha=\alpha\left(X_{3}\right)$, we have $\alpha_{-t}=\alpha$, implying that $(\alpha C)_{-t}=\alpha C_{-t}$. Since $C_{-t}$ is a linear combination of $1, v,|v|^{2} / 2$, with coefficients depending on $x$, the same occurs for $\alpha C_{-t}$. Therefore $(\alpha C)_{-t}$ is an invariant of $Q$ for any $t \in \mathbb{R}$, saying that $\alpha C$ is an invariant of $\langle Q\rangle$, cf. Proposition 4.2.

2. Let $G=G(X, V)$ be a presence density. For any continuous, compactly supported function $\alpha=\alpha\left(X_{3}\right)$, we know that $\alpha C$ is an invariant of $\langle Q\rangle$ and thus

$$
0=\int_{\mathbb{R}^{3}} \int_{\mathbb{R}^{3}}\langle Q\rangle(G) \alpha\left(X_{3}\right) C \mathrm{~d} V \mathrm{~d} X=\int_{\mathbb{R}} \alpha\left(X_{3}\right)\left(\int_{\mathbb{R}^{2}} \int_{\mathbb{R}^{3}}\langle Q\rangle(G) C \mathrm{~d} V \mathrm{~d} \bar{X}\right) \mathrm{d} X_{3}
$$

saying that

$$
\int_{\mathbb{R}^{2}} \int_{\mathbb{R}^{3}}\langle Q\rangle(G) C(X, V) \mathrm{d} V \mathrm{~d} \bar{X}=0, \quad X_{3} \in \mathbb{R} .
$$

3. Let us consider $X_{3}^{\star} \in \mathbb{R}$ and $\alpha^{\star}=\alpha\left(X_{3}^{\star}\right) \geq 0$. The traces of the presence densities $\alpha F$ and $\alpha^{\star} F$ coincide at $X_{3}=X_{3}^{\star}$. As $Q$ is local in space, we deduce by Proposition 4.1 that $\langle Q\rangle$ is local with respect to the parallel space coordinate, implying that the traces of $\langle Q\rangle(\alpha F)$ and $\langle Q\rangle\left(\alpha^{\star} F\right)$ coincide at $X_{3}=X_{3}^{\star}$. Thanks to the homogeneity of $Q$ we have

$$
\begin{aligned}
\langle Q\rangle\left(\alpha^{\star} F\right) & =\frac{1}{T_{c}} \int_{0}^{T_{c}} Q\left(\alpha^{\star} F_{-t}\right)_{t} \mathrm{~d} t \\
& =\frac{1}{T_{c}} \int_{0}^{T_{c}}\left(\alpha^{\star}\right)^{d} Q\left(F_{-t}\right)_{t} \mathrm{~d} t \\
& =\left(\alpha^{\star}\right)^{d}\langle Q\rangle(F)
\end{aligned}
$$

and thus we obtain for any $X_{3}^{\star} \in \mathbb{R},(\bar{X}, V) \in \mathbb{R}^{5}$

$$
\langle Q\rangle(\alpha F)\left(\bar{X}, X_{3}^{\star}, V\right)=\left(\alpha^{\star}\right)^{d}\langle Q\rangle(F)\left(\bar{X}, X_{3}^{\star}, V\right)=\left(\alpha\left(X_{3}^{\star}\right)\right)^{d}\langle Q\rangle(F)\left(\bar{X}, X_{3}^{\star}, V\right) .
$$


In particular, if $F$ is an equilibrium of $\langle Q\rangle$, then $\alpha F$ is also an equilibrium of $\langle Q\rangle$. Let us establish (21), for any presence density $F$. Thanks to (19) we deduce

$$
\begin{aligned}
\int_{\mathbb{R}^{3}} \int_{\mathbb{R}^{3}} \ln F(X, V)\langle Q\rangle(F) \mathrm{d} V \mathrm{~d} X & =\int_{\mathbb{R}^{3}} \int_{\mathbb{R}^{3}} \ln F(X, V) \frac{1}{T_{c}} \int_{0}^{T_{c}} Q\left(F_{-t}\right)_{t} \mathrm{~d} t \mathrm{~d} V \mathrm{~d} X \\
& =\frac{1}{T_{c}} \int_{0}^{T_{c}} \int_{\mathbb{R}^{3}} \int_{\mathbb{R}^{3}} \ln F Q\left(F_{-t}\right)_{t} \mathrm{~d} V \mathrm{~d} X \mathrm{~d} t \\
& =\frac{1}{T_{c}} \int_{0}^{T_{c}} \int_{\mathbb{R}^{3}} \int_{\mathbb{R}^{3}} \ln F_{-t} Q\left(F_{-t}\right) \mathrm{d} v \mathrm{~d} x \mathrm{~d} t \leq 0 .
\end{aligned}
$$

For any function $\alpha=\alpha\left(X_{3}\right)>0$ we have

$$
\int_{\mathbb{R}^{3}} \int_{\mathbb{R}^{3}}\left[\ln \alpha\langle Q\rangle(\alpha F)+\ln F \alpha^{d}\langle Q\rangle(F)\right] \mathrm{d} V \mathrm{~d} X=\int_{\mathbb{R}^{3}} \int_{\mathbb{R}^{3}} \ln (\alpha F)\langle Q\rangle(\alpha F) \mathrm{d} V \mathrm{~d} X \leq 0 .
$$

As $(\ln \alpha)_{-t}=\ln \alpha$ is an invariant of $Q$, for any $t \in \mathbb{R}$, we deduce by Proposition 4.2 that $\ln \alpha$ is an invariant of $\langle Q\rangle$

$$
\int_{\mathbb{R}^{3}} \int_{\mathbb{R}^{3}} \ln \alpha\left(X_{3}\right)\langle Q\rangle(\alpha F) \mathrm{d} V \mathrm{~d} X=0
$$

Therefore (22) becomes

$$
\int_{\mathbb{R}} \alpha\left(X_{3}\right)^{d} \int_{\mathbb{R}^{2}} \int_{\mathbb{R}^{3}} \ln F\langle Q\rangle(F) \mathrm{d} V \mathrm{~d} \bar{X} \mathrm{~d} X_{3} \leq 0
$$

implying that

$$
\int_{\mathbb{R}^{2}} \int_{\mathbb{R}^{3}} \ln F\langle Q\rangle(F) \mathrm{d} V \mathrm{~d} \bar{X} \leq 0, \quad X_{3} \in \mathbb{R}
$$

Remark 4.3 The Fokker-Planck-Landau operator is homogeneous of degree 2.

In the sequel we determine explicitly the invariants of $\langle Q\rangle$.

Proposition 4.4 Let $Q$ be an operator verifying the statements 1. and 2. of Theorem 4.1, whose equilibria are the Maxwellians. Let $C$ be an invariant of $\langle Q\rangle$. In particular we know by Proposition 4.2 that $C$ is an invariant of $Q$ and therefore there are two functions $\alpha=\alpha(X), \gamma=\gamma(X)$ and a vector field $\beta=\beta(X)$ such that

$$
C(X, V)=\alpha(X) \frac{|V|^{2}}{2}+\beta(X) \cdot V+\gamma(X), \quad(X, V) \in \mathbb{R}^{3} \times \mathbb{R}^{3} .
$$

1. The functions $\alpha$ and $\beta_{3}$ depend only on the parallel space coordinate.

2. There are a function $\delta=\delta\left(X_{3}\right)$ and a vector field $\bar{\zeta}=\bar{\zeta}\left(X_{3}\right)$ such that

$$
\bar{\beta}(X)=\delta\left(X_{3}\right)^{\perp} \bar{X}+\bar{\zeta}\left(X_{3}\right), \quad X \in \mathbb{R}^{3} .
$$


3. There are a function $\eta=\eta\left(X_{3}\right)$ and a vector field $\overline{\tilde{\zeta}}=\overline{\tilde{\zeta}}\left(X_{3}\right)$ such that

$$
\gamma(X)=\eta\left(X_{3}\right)+\left[\overline{\tilde{\zeta}}\left(X_{3}\right)+\omega^{\perp} \bar{\zeta}\left(X_{3}\right)\right] \cdot \bar{X}-\omega \delta\left(X_{3}\right) \frac{|\bar{X}|^{2}}{2}, \quad X \in \mathbb{R}^{3} .
$$

4. For any fixed $X_{3} \in \mathbb{R}, C\left(\cdot, \cdot, X_{3}, \cdot, \cdot, \cdot\right)$ is a linear combination of

$$
1, \bar{X}+\frac{{ }^{\perp} \bar{V}}{\omega}, \quad V, \frac{|V|^{2}}{2},\left|\bar{X}+\frac{\perp \bar{V}}{\omega}\right|^{2}-\frac{|\bar{V}|^{2}}{\omega^{2}}
$$

whose coefficients depend on $X_{3}$. Conversely, any linear combination of this kind is an invariant of $\langle Q\rangle$.

\section{Proof.}

1. By Lemma 4.1 we deduce that $b \cdot \nabla_{X, V} C$ is an invariant of $\langle Q\rangle$, and thus of $Q$, cf. Proposition 4.2. There are two functions $\tilde{\alpha}=\tilde{\alpha}(X), \tilde{\gamma}=\tilde{\gamma}(X)$ and a vector field $\tilde{\beta}=\tilde{\beta}(X)$ such that

$$
b \cdot \nabla_{X, V} C=\tilde{\alpha}(X) \frac{|V|^{2}}{2}+\tilde{\beta} \cdot V+\tilde{\gamma}(X), \quad(X, V) \in \mathbb{R}^{3} \times \mathbb{R}^{3}
$$

implying that

$\left(\bar{V} \cdot \nabla_{\bar{X}} \alpha\right) \frac{|V|^{2}}{2}+\left(\bar{V} \cdot \nabla_{\bar{X}}\right) \beta \cdot V+\omega \bar{\beta}(X) \cdot{ }^{\perp} \bar{V}+\bar{V} \cdot \nabla_{\bar{X}} \gamma=\tilde{\alpha}(X) \frac{|V|^{2}}{2}+\tilde{\beta}(X) \cdot V+\tilde{\gamma}(X)$

or equivalently

$$
\begin{gathered}
\bar{V} \cdot \nabla_{\bar{X}} \alpha=0 \\
\left(\bar{V} \cdot \nabla_{\bar{X}}\right) \beta \cdot V=\tilde{\alpha}(X) \frac{|V|^{2}}{2} \\
-\omega^{\perp} \bar{\beta}+\nabla_{\bar{X}} \gamma=\overline{\tilde{\beta}}, \quad \tilde{\beta}_{3}=0 \\
\tilde{\gamma}=0 .
\end{gathered}
$$

The equality (23) implies that the function $\alpha$ depends only on $X_{3}$. We established that the coefficient of the term $|V|^{2} / 2$ in any invariant of $\langle Q\rangle$ depends only on $X_{3}$. As $b \cdot \nabla_{X, V} C$ is also an invariant of $\langle Q\rangle$, we deduce that $\tilde{\alpha}$ depends only on $X_{3}$. By taking $V={ }^{t}(0,0,1)$ in $(24)$ we obtain $\tilde{\alpha}=0$, implying that

$$
\left(\left(\bar{V} \cdot \nabla_{\bar{X}}\right) \bar{\beta}\right) \cdot \bar{V}+\left(\bar{V} \cdot \nabla_{\bar{X}} \beta_{3}\right) V_{3}=0, \quad \bar{V} \in \mathbb{R}^{2}, \quad V_{3} \in \mathbb{R} .
$$

Therefore $\nabla_{\bar{X}} \beta_{3}=0$ and the matrix $\partial_{\bar{X}} \bar{\beta}$ is anti-symmetric

$$
\partial_{X_{1}} \beta_{1}=0, \quad \partial_{X_{1}} \beta_{2}+\partial_{X_{2}} \beta_{1}=0, \quad \partial_{X_{2}} \beta_{2}=0 .
$$

2. It is easily seen that

$$
\begin{aligned}
\nabla_{\bar{X}} \partial_{X_{1}} \beta_{2} & ={ }^{t}\left(\partial_{X_{1}}^{2} \beta_{2}, \partial_{X_{2}} \partial_{X_{1}} \beta_{2}\right)={ }^{t}\left(-\partial_{X_{1}} \partial_{X_{2}} \beta_{1}, \partial_{X_{2}} \partial_{X_{1}} \beta_{2}\right) \\
& ={ }^{t}\left(-\partial_{X_{2}} \partial_{X_{1}} \beta_{1}, \partial_{X_{1}} \partial_{X_{2}} \beta_{2}\right)={ }^{t}(0,0)
\end{aligned}
$$


and therefore, there is a function $\delta=\delta\left(X_{3}\right)$ such that

$$
\partial_{X_{2}} \beta_{1}=-\partial_{X_{1}} \beta_{2}=\delta\left(X_{3}\right) \text {. }
$$

Notice that

$$
\begin{aligned}
& \nabla_{\bar{X}}\left(\beta_{1}-X_{2} \delta\left(X_{3}\right)\right)={ }^{t}\left(\partial_{X_{1}} \beta_{1}, \partial_{X_{2}} \beta_{1}-\delta\left(X_{3}\right)\right)={ }^{t}(0,0) \\
& \nabla_{\bar{X}}\left(\beta_{2}+X_{1} \delta\left(X_{3}\right)\right)={ }^{t}\left(\partial_{X_{1}} \beta_{2}+\delta\left(X_{3}\right), \partial_{X_{2}} \beta_{2}\right)={ }^{t}(0,0)
\end{aligned}
$$

implying that there is a vector field $\bar{\zeta}=\bar{\zeta}\left(X_{3}\right)$ such that

$$
\bar{\beta}(X)=\delta\left(X_{3}\right)^{\perp} \bar{X}+\bar{\zeta}\left(X_{3}\right) .
$$

As $b \cdot \nabla_{X, V} C$ is also an invariant of $\langle Q\rangle$, there are a function $\tilde{\delta}=\tilde{\delta}\left(X_{3}\right)$ and a vector

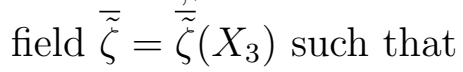

$$
\overline{\tilde{\beta}}(X)=\tilde{\delta}\left(X_{3}\right)^{\perp} \bar{X}+\overline{\tilde{\zeta}}\left(X_{3}\right) .
$$

3. In order to determine the function $\gamma$ we appeal to (25)

$$
\omega\left[\delta\left(X_{3}\right){ }^{\perp} \bar{X}+\bar{\zeta}\left(X_{3}\right)\right]+{ }^{\perp} \nabla_{\bar{X}} \gamma=\omega \bar{\beta}+{ }^{\perp} \nabla_{\bar{X}} \gamma={ }^{\perp} \overline{\tilde{\beta}}=-\tilde{\delta}\left(X_{3}\right) \bar{X}+{ }^{\perp} \overline{\tilde{\zeta}}\left(X_{3}\right) .
$$

By taking the divergence with respect to $\bar{X}$, we obtain the compatibility condition $\tilde{\delta}=0$ and

$$
\nabla_{\bar{X}} \gamma=\overline{\tilde{\zeta}}\left(X_{3}\right)+\omega\left[{ }^{\perp} \bar{\zeta}\left(X_{3}\right)-\delta\left(X_{3}\right) \bar{X}\right]
$$

Therefore there is a function $\eta=\eta\left(X_{3}\right)$ such that

$$
\gamma(X)=\eta\left(X_{3}\right)+\left[\overline{\tilde{\zeta}}\left(X_{3}\right)+\omega^{\perp} \bar{\zeta}\left(X_{3}\right)\right] \cdot \bar{X}-\omega \delta\left(X_{3}\right) \frac{|\bar{X}|^{2}}{2} .
$$

4. Combining the previous three statements, we deduce that

$$
\begin{aligned}
C(X, V) & =\alpha\left(X_{3}\right) \frac{|V|^{2}}{2}+\beta_{3}\left(X_{3}\right) V_{3}+\left[\delta\left(X_{3}\right)^{\perp} \bar{X}+\bar{\zeta}\left(X_{3}\right)\right] \cdot \bar{V}+\eta\left(X_{3}\right) \\
& +\left[\overline{\tilde{\zeta}}\left(X_{3}\right)+\omega^{\perp} \bar{\zeta}\left(X_{3}\right)\right] \cdot \bar{X}-\omega \delta\left(X_{3}\right) \frac{|\bar{X}|^{2}}{2} \\
& =\eta\left(X_{3}\right)+\left[\overline{\tilde{\zeta}}\left(X_{3}\right)+\omega^{\perp} \bar{\zeta}\left(X_{3}\right)\right] \cdot\left(\bar{X}+\frac{\perp \bar{V}}{\omega}\right)+{ }^{\perp} \overline{\tilde{\zeta}}\left(X_{3}\right) \cdot \frac{\bar{V}}{\omega}+\beta_{3}\left(X_{3}\right) V_{3} \\
& +\alpha\left(X_{3}\right) \frac{|V|^{2}}{2}-\omega \frac{\delta\left(X_{3}\right)}{2}\left(\left|\bar{X}+\frac{\perp \bar{V}}{\omega}\right|^{2}-\frac{|\bar{V}|^{2}}{\omega^{2}}\right)
\end{aligned}
$$

Conversely, we claim that any function given by (26) is an invariant of $\langle Q\rangle$. Obviously, the functions $\eta\left(X_{3}\right), \beta_{3}\left(X_{3}\right) V_{3}$,

$$
\left[\overline{\tilde{\zeta}}\left(X_{3}\right)+\omega^{\perp} \bar{\zeta}\left(X_{3}\right)\right] \cdot\left(\bar{X}+\frac{{ }^{\perp} \bar{V}}{\omega}\right), \alpha\left(X_{3}\right) \frac{|V|^{2}}{2}, \omega \frac{\delta\left(X_{3}\right)}{2}\left(\left|\bar{X}+\frac{\perp \bar{V}}{\omega}\right|^{2}-\frac{|\bar{V}|^{2}}{\omega^{2}}\right)
$$

are invariants of $Q$, and are also left invariant by the flow of $b \cdot \nabla_{X, V}$. By Proposition 4.2 we deduce that all the above expressions are invariants of $\langle Q\rangle$. It remains to justify that $\perp \overline{\tilde{\zeta}}\left(X_{3}\right) \cdot \bar{V}$ is also an invariant of $\langle Q\rangle$. For any $t \in \mathbb{R}$, the function

$$
\left(\perp \overline{\tilde{\zeta}}\left(X_{3}\right) \cdot \bar{V}\right)_{-t}=\perp \overline{\tilde{\zeta}}\left(X_{3}\right) \cdot \mathcal{R}(\omega t) \bar{V}
$$

is an invariant of $Q$, and thus $\perp \overline{\tilde{\zeta}}\left(X_{3}\right) \cdot \bar{V}$ is an invariant of $\langle Q\rangle$, cf. Proposition 4.2. 
Once that the invariants of $\langle Q\rangle$ have been identified, the equilibria of $\langle Q\rangle$ follow thanks to Remark 4.2. Any equilibrium of $\langle Q\rangle$ writes $F(X, V)=e^{C(X, V)}$, where $C$ is an invariant of $\langle Q\rangle$. Therefore, for any fixed $X_{3} \in \mathbb{R}, \ln F\left(\cdot, \cdot, X_{3}, \cdot, \cdot, \cdot\right)$ has the form (26).

For any dimension $N$ and temperature $T>0$, we denote by $\mathcal{M}_{T}^{N}$ the Maxwellian

$$
\mathcal{M}_{T}^{N}(w)=\frac{1}{(2 \pi T)^{N / 2}} \exp \left(-\frac{|w|^{2}}{2 T}\right), w \in \mathbb{R}^{N}
$$

For simplicity, we will write $\mathcal{M}_{T}(w)$, the dimension of the phase space being that of the Maxwellian argument.

Proposition 4.5 Let $Q$ be an operator verifying the statements 1. and 2. of Theorem 4.1, whose equilibria are the Maxwellians. Let $F=F(X, V)>0$ be an equilibrium of $\langle Q\rangle$ such that the following moments are well defined

$$
\int_{\mathbb{R}^{2}} \int_{\mathbb{R}^{3}} F\left\{1, V, \omega \bar{X}+{ }^{\perp} \bar{V}, \frac{|V|^{2}}{2}, \frac{\left|\omega \bar{X}+{ }^{\perp} \bar{V}\right|^{2}-|\bar{V}|^{2}}{2}\right\} \mathrm{d} V \mathrm{~d} \bar{X}, \quad X_{3} \in \mathbb{R} .
$$

Then there are $n=n\left(X_{3}\right): \mathbb{R} \rightarrow \mathbb{R}_{+}, \theta=\theta\left(X_{3}\right): \mathbb{R} \rightarrow \mathbb{R}_{+}, \mu=\mu\left(X_{3}\right): \mathbb{R} \rightarrow \mathbb{R}_{+}$, $U=U\left(X_{3}\right): \mathbb{R} \rightarrow \mathbb{R}^{3}$ and $\bar{Y}=\bar{Y}\left(X_{3}\right): \mathbb{R} \rightarrow \mathbb{R}^{2}$ verifying $n>0, \mu>\theta>0$ such that

$$
F(X, V)=\omega^{2} n\left(X_{3}\right) \mathcal{M}_{\frac{\mu \theta}{\mu-\theta}}(\bar{V}-\bar{U}) \mathcal{M}_{\theta}\left(V_{3}-U_{3}\right) \mathcal{M}_{\mu}\left(\omega \bar{X}+{ }^{\perp} \bar{V}-\bar{Y}\right) .
$$

The functions $n>0, \mu>\theta>0$ and the vector fields $U, \bar{Y}$ are uniquely determined by

$$
\begin{gathered}
\int_{\mathbb{R}^{2}} \int_{\mathbb{R}^{3}} F(X, V) \mathrm{d} V \mathrm{~d} \bar{X}=n\left(X_{3}\right), \quad \int_{\mathbb{R}^{2}} \int_{\mathbb{R}^{3}} F(X, V) V \mathrm{~d} V \mathrm{~d} \bar{X}=n\left(X_{3}\right) U\left(X_{3}\right) \\
\int_{\mathbb{R}^{2}} \int_{\mathbb{R}^{3}} F(X, V)\left(\omega \bar{X}+{ }^{\perp} \bar{V}\right) \mathrm{d} V \mathrm{~d} \bar{X}=n\left(X_{3}\right) \bar{Y}\left(X_{3}\right) \\
\int_{\mathbb{R}^{2}} \int_{\mathbb{R}^{3}} F(X, V) \frac{|V-U|^{2}}{2} \mathrm{~d} V \mathrm{~d} \bar{X}=n\left(X_{3}\right)\left(\frac{\mu \theta}{\mu-\theta}+\frac{\theta}{2}\right) \\
\int_{\mathbb{R}^{2}} \int_{\mathbb{R}^{3}} F(X, V) \frac{\left|\omega \bar{X}+{ }^{\perp} \bar{V}-\bar{Y}\right|^{2}-|\bar{V}-\bar{U}|^{2}}{2} \mathrm{~d} V \mathrm{~d} \bar{X}=n\left(X_{3}\right)\left(\mu-\frac{\mu \theta}{\mu-\theta}\right) .
\end{gathered}
$$

Proof. By Proposition 4.4, up to a factor (depending on $X_{3}$ ), the equilibrium $F$ writes

$$
\begin{aligned}
& \exp \left(-\frac{\left|V-u\left(X_{3}\right)\right|^{2}}{2 \theta\left(X_{3}\right)}\right) \exp \left(-\frac{\left|\omega \bar{X}+{ }^{\perp} \bar{V}-\bar{Y}\left(X_{3}\right)\right|^{2}-|\bar{V}|^{2}}{2 \mu\left(X_{3}\right)}\right) \\
& =\exp \left(-\frac{\left|\bar{V}-\frac{\mu}{\mu-\theta} \bar{u}\left(X_{3}\right)\right|^{2}}{2 \frac{\mu \theta}{\mu-\theta}}+\frac{\left|\bar{u}\left(X_{3}\right)\right|^{2}}{2(\mu-\theta)}\right) \exp \left(-\frac{\left(V_{3}-u_{3}\left(X_{3}\right)\right)^{2}}{2 \theta\left(X_{3}\right)}\right) \\
& \times \exp \left(-\frac{\left|\omega \bar{X}+{ }^{\perp} \bar{V}-\bar{Y}\left(X_{3}\right)\right|^{2}}{2 \mu\left(X_{3}\right)}\right)
\end{aligned}
$$


and thus

$$
F(X, V)=\omega^{2} n\left(X_{3}\right) \mathcal{M}_{\frac{\mu \theta}{\mu-\theta}}\left(\bar{V}-\frac{\mu}{\mu-\theta} \bar{u}\right) \mathcal{M}_{\theta}\left(V_{3}-u_{3}\right) \mathcal{M}_{\mu}\left(\omega \bar{X}+{ }^{\perp} \bar{V}-\bar{Y}\right) .
$$

By introducing the notation $U=\left(\frac{\mu}{\mu-\theta} \bar{u}, u_{3}\right)$, we obtain

$$
F(X, V)=\omega^{2} n\left(X_{3}\right) \mathcal{M}_{\frac{\mu \theta}{\mu-\theta}}(\bar{V}-\bar{U}) \mathcal{M}_{\theta}\left(V_{3}-U_{3}\right) \mathcal{M}_{\mu}\left(\omega \bar{X}+{ }^{\perp} \bar{V}-\bar{Y}\right) .
$$

It is easily seen that

$$
\begin{aligned}
\int_{\mathbb{R}^{2}} \int_{\mathbb{R}^{3}} F \mathrm{~d} V \mathrm{~d} \bar{X} & =n \int_{\mathbb{R}^{3}} \mathcal{M}_{\frac{\mu \theta}{\mu-\theta}}(\bar{V}-\bar{U}) \mathcal{M}_{\theta}\left(V_{3}-U_{3}\right) \int_{\mathbb{R}^{2}} \mathcal{M}_{\mu}\left(\omega \bar{X}+{ }^{\perp} \bar{V}-\bar{Y}\right) \mathrm{d}(\omega \bar{X}) \mathrm{d} V \\
& =n \int_{\mathbb{R}^{2}} \mathcal{M}_{\frac{\mu \theta}{\mu-\theta}}(\bar{V}-\bar{U}) \mathrm{d} \bar{V} \int_{\mathbb{R}} \mathcal{M}_{\theta}\left(V_{3}-U_{3}\right) \mathrm{d} V_{3}=n
\end{aligned}
$$

and

$$
\begin{aligned}
& \int_{\mathbb{R}^{2}} \int_{\mathbb{R}^{3}} F \bar{V} \mathrm{~d} V \mathrm{~d} \bar{X}=n \int_{\mathbb{R}^{3}} \mathcal{M}_{\frac{\mu \theta}{\mu-\theta}}(\bar{V}-\bar{U}) \mathcal{M}_{\theta}\left(V_{3}-U_{3}\right) \bar{V} \int_{\mathbb{R}^{2}} \mathcal{M}_{\mu}\left(\omega \bar{X}+{ }^{\perp} \bar{V}-\bar{Y}\right) \mathrm{d}(\omega \bar{X}) \mathrm{d} V \\
&=n\left(X_{3}\right) \bar{U}\left(X_{3}\right) \\
& \int_{\mathbb{R}^{2}} \int_{\mathbb{R}^{3}} F V_{3} \mathrm{~d} V \mathrm{~d} \bar{X}=n\left(X_{3}\right) U_{3}\left(X_{3}\right), \quad \int_{\mathbb{R}^{2}} \int_{\mathbb{R}^{3}} F\left(\omega \bar{X}+{ }^{\perp} \bar{V}\right) \mathrm{d} V \mathrm{~d} \bar{X}=n\left(X_{3}\right) \bar{Y}\left(X_{3}\right) .
\end{aligned}
$$

We concentrate now on the quadratic moments. Thanks to the formula

$$
\int_{\mathbb{R}^{N}} \mathcal{M}_{T}^{N}(w) \frac{|w|^{2}}{2} \mathrm{~d} w=\frac{N}{2} T
$$

we obtain

$$
\begin{aligned}
\int_{\mathbb{R}^{2}} \int_{\mathbb{R}^{3}} F \frac{|V-U|^{2}}{2} \mathrm{~d} V \mathrm{~d} \bar{X}=\int_{\mathbb{R}^{2}} \int_{\mathbb{R}^{3}} F \frac{|\bar{V}-\bar{U}|^{2}}{2} \mathrm{~d} V \mathrm{~d} \bar{X}+\int_{\mathbb{R}^{2}} \int_{\mathbb{R}^{3}} F \frac{\left(V_{3}-U_{3}\right)^{2}}{2} \mathrm{~d} V \mathrm{~d} \bar{X} \\
\quad=n \int_{\mathbb{R}^{2}} \mathcal{M}_{\frac{\mu \theta}{\mu-\theta}}(\bar{V}-\bar{U}) \frac{|\bar{V}-\bar{U}|^{2}}{2} \mathrm{~d} \bar{V}+\int_{\mathbb{R}} \mathcal{M}_{\theta}\left(V_{3}-U_{3}\right) \frac{\left(V_{3}-U_{3}\right)^{2}}{2} \mathrm{~d} V_{3} \\
\quad=n\left(X_{3}\right)\left(\frac{\mu \theta}{\mu-\theta}+\frac{\theta}{2}\right)
\end{aligned}
$$

and

$$
\int_{\mathbb{R}^{2}} \int_{\mathbb{R}^{3}} F(X, V) \frac{\left|\omega \bar{X}+{ }^{\perp} \bar{V}-\bar{Y}\right|^{2}-|\bar{V}-\bar{U}|^{2}}{2} \mathrm{~d} V \mathrm{~d} \bar{X}=n\left(X_{3}\right)\left(\mu-\frac{\mu \theta}{\mu-\theta}\right) .
$$

The concentration $n\left(X_{3}\right)$, the velocity $U\left(X_{3}\right)$ and the Larmor center $\bar{Y}\left(X_{3}\right) / \omega$ express easily in terms of the moments $\int_{\mathbb{R}^{2}} \int_{\mathbb{R}^{3}} F\left\{1, V, \omega \bar{X}+{ }^{\perp} \bar{V}\right\} \mathrm{d} V \mathrm{~d} \bar{X}$. It remains to determine $\theta, \mu$ thanks to

$$
n K=\int_{\mathbb{R}^{2}} \int_{\mathbb{R}^{3}} F \frac{|V-U|^{2}}{2} \mathrm{~d} V \mathrm{~d} \bar{X}, \quad n G=\int_{\mathbb{R}^{2}} \int_{\mathbb{R}^{3}} F \frac{|\omega \bar{X}+\perp \bar{V}-\bar{Y}|^{2}-|\bar{V}-\bar{U}|^{2}}{2} \mathrm{~d} V \mathrm{~d} \bar{X} .
$$


Notice that we have $n\left(X_{3}\right)\left(K\left(X_{3}\right)+G\left(X_{3}\right)\right)=\int_{\mathbb{R}^{2}} \int_{\mathbb{R}^{3}} F \frac{\left|\omega \bar{X}+{ }^{\perp} \bar{V}-\bar{Y}\right|^{2}+\left(V_{3}-U_{3}\right)^{2}}{2} \mathrm{~d} V \mathrm{~d} \bar{X}>0, \quad X_{3} \in \mathbb{R}$.

We have to solve the system

$$
\frac{\mu \theta}{\mu-\theta}+\frac{\theta}{2}=K, \quad \mu-\frac{\mu \theta}{\mu-\theta}=G
$$

We search for $\nu:=\frac{\mu}{\theta}>1$ verifying

$$
\frac{G}{K}=\frac{\mu-\frac{\mu \theta}{\mu-\theta}}{\frac{\mu \theta}{\mu-\theta}+\frac{\theta}{2}}=\frac{\nu-\frac{\nu}{\nu-1}}{\frac{\nu}{\nu-1}+\frac{1}{2}}=2 \nu \frac{\nu-2}{3 \nu-1}
$$

or equivalently

$$
2(\nu-1)^{2}-3 S(\nu-1)-2(S+1)=0, \quad S=\frac{G}{K}>-1 .
$$

The only solution $\nu>1$ is $\nu=1+\frac{3 S+\sqrt{9 S^{2}+16(S+1)}}{4}$. Combining to the relation $\frac{\theta}{2}+\mu=$ $K+G$, we obtain

$$
\theta=\frac{K+G}{\frac{1}{2}+\nu}>0, \quad \mu=\nu \theta=\nu \frac{K+G}{\frac{1}{2}+\nu}>\theta>0 .
$$

\section{The Euler gyro-kinetic equations}

We are ready to write the fluid model corresponding to the limit system (5), when $\tau \searrow 0$. Clearly we have

$$
\begin{aligned}
(\varphi(t / \varepsilon) a(t))(X, V) & =\partial \mathcal{Z}(-t / \varepsilon ; \mathcal{Z}(t / \varepsilon ; X, V)) a(t, \mathcal{Z}(t / \varepsilon ; X, V)) \\
& =V_{3} \partial_{X_{3}}+\left(I_{2}-\mathcal{R}(\omega t / \varepsilon)\right) \frac{\perp \bar{E}}{B}(t, \mathcal{X}(t / \varepsilon ; X, V)) \cdot \nabla_{\bar{X}} \\
& +\frac{q}{m} \mathcal{R}(\omega t / \varepsilon) \bar{E}(t, \mathcal{X}(t / \varepsilon ; X, V)) \cdot \nabla_{\bar{V}}+\frac{q}{m} E_{3}(t, \mathcal{X}(t / \varepsilon ; X, V)) \partial_{V_{3}}
\end{aligned}
$$

and thus the average with respect to the fast dynamics writes

$$
\begin{aligned}
& \langle a(t)\rangle(X, V)=\frac{1}{T_{c}} \int_{0}^{T_{c}}(\varphi(s) a(t))(X, V) \mathrm{d} s \\
& =\frac{1}{T_{c}} \int_{0}^{T_{c}} t\left(\left(I_{2}-\mathcal{R}(\omega s)\right) \frac{\perp \bar{E}}{B}(t, \mathcal{X}(s ; X, V)), V_{3}, \frac{q}{m}\left(\mathcal{R}(\omega s) \bar{E}, E_{3}\right)(t, \mathcal{X}(s ; X, V))\right) \mathrm{d} s .
\end{aligned}
$$

When the electric field derives from a potential i.e., $E(t)=-\nabla_{x} \Phi(t)$ it is easily seen that

$$
\frac{\mathrm{d}}{\mathrm{d} s} \Phi(t, \mathcal{X}(s ; X, V))=-\mathcal{R}(\omega s) \bar{E}(t, \mathcal{X}(s ; X, V)) \cdot \bar{V}
$$


implying that $\frac{1}{T_{c}} \int_{0}^{T_{c}} \mathcal{R}(\omega s) \frac{\bar{E}}{B}(t, \mathcal{X}(s ; X, V)) \mathrm{d} s=0$ and therefore

$$
\begin{aligned}
\langle a(t)\rangle(X, V) & =\frac{1}{T_{c}} \int_{0}^{T_{c}} \frac{\perp E}{B}(t, \mathcal{X}(s ; X, V)) \mathrm{d} s \cdot \nabla_{\bar{X}}+V_{3} \partial_{X_{3}} \\
& +\frac{1}{T_{c}} \int_{0}^{T_{c}} \frac{q}{m} E_{3}(t, \mathcal{X}(s ; X, V)) \mathrm{d} s \partial_{V_{3}} .
\end{aligned}
$$

For simplicity we assume that at any time $t$ the electric field $E(t)$ is uniform (the arguments apply as well for non uniform electric fields, but the computations are more complicated and involve additional terms). In this case, the average vector field simply writes

$$
\langle a(t)\rangle(X, V) \cdot \nabla_{X, V}=\frac{\perp \bar{E}(t)}{B} \cdot \nabla_{\bar{X}}+V_{3} \partial_{X_{3}}+\frac{q}{m} E_{3}(t) \partial_{V_{3}}
$$

and the equation (5) becomes

$\partial_{t} F^{\tau}+\frac{\perp \bar{E}(t)}{B} \cdot \nabla_{\bar{X}} F^{\tau}+V_{3} \partial_{X_{3}} F^{\tau}+\frac{q}{m} E_{3}(t) \partial_{V_{3}} F^{\tau}=\frac{1}{\tau}\langle Q\rangle\left(F^{\tau}\right), \quad(t, X, V) \in \mathbb{R}_{+} \times \mathbb{R}^{3} \times \mathbb{R}^{3}$.

At any time $t$, the limit presence density $F(t, \cdot, \cdot)=\lim _{\tau \backslash{ }_{0}} F^{\tau}(t, \cdot, \cdot)$ is an equilibrium of $\langle Q\rangle$, and therefore there are $n\left(t, X_{3}\right), \theta\left(t, X_{3}\right), \mu\left(t, X_{3}\right), U\left(t, X_{3}\right), \bar{Y}\left(t, X_{3}\right)$ such that

$$
F(t, X, V)=\omega^{2} n\left(t, X_{3}\right) \mathcal{M}_{\frac{\mu \theta}{\mu-\theta}}(\bar{V}-\bar{U}) \mathcal{M}_{\theta}\left(V_{3}-U_{3}\right) \mathcal{M}_{\mu}\left(\omega \bar{X}+{ }^{\perp} \bar{V}-\bar{Y}\right) .
$$

Recall that for any invariant of $\langle Q\rangle$ we have, thanks to Proposition 4.3

$$
\int_{\mathbb{R}^{2}} \int_{\mathbb{R}^{3}}\langle Q\rangle(G) C(X, V) \mathrm{d} V \mathrm{~d} \bar{X}=0, \quad X_{3} \in \mathbb{R}
$$

for any presence density $G=G(X, V)$. We deduce that the limit presence density satisfies

$$
\begin{aligned}
& \partial_{t} \int_{\mathbb{R}^{2}} \int_{\mathbb{R}^{3}} F(t, X, V) C(X, V) \mathrm{d} V \mathrm{~d} \bar{X}+\partial_{X_{3}} \int_{\mathbb{R}^{2}} \int_{\mathbb{R}^{3}} V_{3} F(t, X, V) C(X, V) \mathrm{d} V \mathrm{~d} \bar{X} \\
& -\int_{\mathbb{R}^{2}} \int_{\mathbb{R}^{3}} \frac{\perp \bar{E}(t)}{B} \cdot \nabla_{\bar{X}} C F(t, X, V) \mathrm{d} V \mathrm{~d} \bar{X}-\int_{\mathbb{R}^{2}} \int_{\mathbb{R}^{3}} \frac{q}{m} E_{3}(t) \partial_{V_{3}} C F(t, X, V) \mathrm{d} V \mathrm{~d} \bar{X}=0
\end{aligned}
$$

for any invariant $C$. We are done if we consider the invariants

$$
1, \quad V, \quad \omega \bar{X}+{ }^{\perp} \bar{V}, \quad|V|^{2} / 2, \quad\left(\left|\omega \bar{X}+{ }^{\perp} \bar{V}\right|^{2}-|\bar{V}|^{2}\right) / 2 .
$$

We justify the fluid model stated in Theorem 1.1.

Proof. (of Theorem 1.1)

The continuity equation follows by using the invariant $C=1$

$$
\partial_{t} \int_{\mathbb{R}^{2}} \int_{\mathbb{R}^{3}} F \mathrm{~d} V \mathrm{~d} \bar{X}+\partial_{X_{3}} \int_{\mathbb{R}^{2}} \int_{\mathbb{R}^{3}} V_{3} F \mathrm{~d} V \mathrm{~d} \bar{X}=\partial_{t} n+\partial_{X_{3}}\left(n U_{3}\right)=0 .
$$

In order to use the invariants $V$ and $\omega \bar{X}+{ }^{\perp} \bar{V}$, we need to compute the moments of $V_{3} V$ and $V_{3}\left(\omega \bar{X}+{ }^{\perp} \bar{V}\right)$. We obtain

$$
\begin{aligned}
\int_{\mathbb{R}^{2}} \int_{\mathbb{R}^{3}} F V_{3} V \mathrm{~d} V \mathrm{~d} \bar{X} & =\int_{\mathbb{R}^{2}} \int_{\mathbb{R}^{3}} F\left(V_{3} \bar{V}, 0\right) \mathrm{d} V \mathrm{~d} \bar{X}+\int_{\mathbb{R}^{2}} \int_{\mathbb{R}^{3}} F\left(0,0,\left(V_{3}\right)^{2}\right) \mathrm{d} V \mathrm{~d} \bar{X} \\
& =n\left(U_{3} \bar{U}, 0\right)+n\left(0,0, \theta+U_{3}^{2}\right)=n\left[U_{3} U+(0,0, \theta)\right]
\end{aligned}
$$




$$
\int_{\mathbb{R}^{2}} \int_{\mathbb{R}^{3}} F V_{3}\left(\omega \bar{X}+{ }^{\perp} \bar{V}\right) \mathrm{d} V \mathrm{~d} \bar{X}=n U_{3} \bar{Y}
$$

implying the balances for the momentum and the Larmor center

$$
\begin{aligned}
\partial_{t} \int_{\mathbb{R}^{2}} \int_{\mathbb{R}^{3}} F V \mathrm{~d} V \mathrm{~d} \bar{X}+\partial_{X_{3}} \int_{\mathbb{R}^{2}} \int_{\mathbb{R}^{3}} F V_{3} V \mathrm{~d} V \mathrm{~d} \bar{X} & =\partial_{t}(n U)+\partial_{X_{3}}\left(n U_{3} U+n^{t}(0,0, \theta)\right) \\
& ={ }^{t}\left(0,0, \frac{q}{m} E_{3}(t) n\right) \\
\partial_{t} \int_{\mathbb{R}^{2}} \int_{\mathbb{R}^{3}} F\left(\omega \bar{X}+{ }^{\perp} \bar{V}\right) \mathrm{d} V \mathrm{~d} \bar{X}+\partial_{X_{3}} \int_{\mathbb{R}^{2}} \int_{\mathbb{R}^{3}} F V_{3}\left(\omega \bar{X}+{ }^{\perp} \bar{V}\right) \mathrm{d} V \mathrm{~d} \bar{X} & =\partial_{t}(n \bar{Y})+\partial_{X_{3}}\left(n U_{3} \bar{Y}\right) \\
& =\frac{q}{m} \perp \bar{E}(t) n .
\end{aligned}
$$

We compute now the moments of the invariants $|V|^{2} / 2,\left(\left|\omega \bar{X}+{ }^{\perp} \bar{V}\right|^{2}-|\bar{V}|^{2}\right) / 2$

$$
\begin{aligned}
\int_{\mathbb{R}^{2}} \int_{\mathbb{R}^{3}} F \frac{|V|^{2}}{2} \mathrm{~d} V \mathrm{~d} \bar{X} & =\int_{\mathbb{R}^{2}} \int_{\mathbb{R}^{3}} F \frac{|V-U|^{2}}{2} \mathrm{~d} V \mathrm{~d} \bar{X}+\int_{\mathbb{R}^{2}} \int_{\mathbb{R}^{3}} F \frac{|U|^{2}}{2} \mathrm{~d} V \mathrm{~d} \bar{X} \\
& =n\left(\frac{\mu \theta}{\mu-\theta}+\frac{\theta}{2}+\frac{|U|^{2}}{2}\right)
\end{aligned}
$$

and

$$
\begin{aligned}
\int_{\mathbb{R}^{2}} \int_{\mathbb{R}^{3}} F & \frac{\left|\omega \bar{X}+{ }^{\perp} \bar{V}\right|^{2}-|\bar{V}|^{2}}{2} \mathrm{~d} V \mathrm{~d} \bar{X} \\
& =\int_{\mathbb{R}^{2}} \int_{\mathbb{R}^{3}} F \frac{\left|\omega \bar{X}+{ }^{\perp} \bar{V}-\bar{Y}\right|^{2}+|\bar{Y}|^{2}-|\bar{V}-\bar{U}|^{2}-|\bar{U}|^{2}}{2} \mathrm{~d} V \mathrm{~d} \bar{X} \\
& =n\left(\mu+\frac{|\bar{Y}|^{2}}{2}-\frac{\mu \theta}{\mu-\theta}-\frac{|\bar{U}|^{2}}{2}\right) .
\end{aligned}
$$

The corresponding fluxes are given by

$$
\begin{aligned}
\int_{\mathbb{R}^{2}} \int_{\mathbb{R}^{3}} F V_{3} \frac{|V|^{2}}{2} \mathrm{~d} V \mathrm{~d} \bar{X} & =\int_{\mathbb{R}^{2}} \int_{\mathbb{R}^{3}} F V_{3} \frac{|\bar{V}|^{2}}{2} \mathrm{~d} V \mathrm{~d} \bar{X}+\int_{\mathbb{R}^{2}} \int_{\mathbb{R}^{3}} F V_{3} \frac{V_{3}^{2}}{2} \mathrm{~d} V \mathrm{~d} \bar{X} \\
& =n U_{3} \int_{\mathbb{R}^{2}} \mathcal{M}_{\frac{\mu \theta}{\mu-\theta}}(\bar{V}-\bar{U}) \frac{|\bar{V}|^{2}}{2} \mathrm{~d} \bar{V}+n \int_{\mathbb{R}} \mathcal{M}_{\theta}\left(V_{3}-U_{3}\right) V_{3} \frac{V_{3}^{2}}{2} \mathrm{~d} V_{3} \\
& =n U_{3}\left(\frac{\mu \theta}{\mu-\theta}+\frac{|\bar{U}|^{2}}{2}\right)+n U_{3}\left(\frac{\theta}{2}+\frac{U_{3}^{2}}{2}\right) \\
& +n \int_{\mathbb{R}} \mathcal{M}_{\theta}\left(V_{3}-U_{3}\right)\left(V_{3}-U_{3}\right) \frac{\left(V_{3}-U_{3}+U_{3}\right)^{2}}{2} \mathrm{~d} V_{3} \\
& =n U_{3}\left(\frac{\mu \theta}{\mu-\theta}+\frac{3 \theta}{2}+\frac{|U|^{2}}{2}\right)
\end{aligned}
$$


and

$$
\int_{\mathbb{R}^{2}} \int_{\mathbb{R}^{3}} F V_{3} \frac{\left|\omega \bar{X}+{ }^{\perp} \bar{V}\right|^{2}-|\bar{V}|^{2}}{2} \mathrm{~d} V \mathrm{~d} \bar{X}=n U_{3}\left(\mu-\frac{\mu \theta}{\mu-\theta}+\frac{|\bar{Y}|^{2}}{2}-\frac{|\bar{U}|^{2}}{2}\right) .
$$

We obtain the equations

$$
\begin{aligned}
& \partial_{t} \int_{\mathbb{R}^{2}} \int_{\mathbb{R}^{3}} F \frac{|V|^{2}}{2} \mathrm{~d} V \mathrm{~d} \bar{X}+\partial_{X_{3}} \int_{\mathbb{R}^{2}} \int_{\mathbb{R}^{3}} F V_{3} \frac{|V|^{2}}{2} \mathrm{~d} V \mathrm{~d} \bar{X} \\
& =\partial_{t}\left[n\left(\frac{\mu \theta}{\mu-\theta}+\frac{\theta}{2}+\frac{|U|^{2}}{2}\right)\right]+\partial_{X_{3}}\left[n U_{3}\left(\frac{\mu \theta}{\mu-\theta}+\frac{3 \theta}{2}+\frac{|U|^{2}}{2}\right)\right]=\frac{q}{m} E_{3}(t) U_{3} n
\end{aligned}
$$

and

$$
\begin{aligned}
& \partial_{t} \int_{\mathbb{R}^{2}} \int_{\mathbb{R}^{3}} F \frac{\left|\omega \bar{X}+{ }^{\perp} \bar{V}\right|^{2}-|\bar{V}|^{2}}{2} \mathrm{~d} V \mathrm{~d} \bar{X}+\partial_{X_{3}} \int_{\mathbb{R}^{2}} \int_{\mathbb{R}^{3}} F V_{3} \frac{\left|\omega \bar{X}+{ }^{\perp} \bar{V}\right|^{2}-|\bar{V}|^{2}}{2} \mathrm{~d} V \mathrm{~d} \bar{X} \\
& =\partial_{t}\left[n\left(\mu+\frac{|\bar{Y}|^{2}}{2}-\frac{\mu \theta}{\mu-\theta}-\frac{|\bar{U}|^{2}}{2}\right)\right]+\partial_{X_{3}}\left[n U_{3}\left(\mu+\frac{|\bar{Y}|^{2}}{2}-\frac{\mu \theta}{\mu-\theta}-\frac{|\bar{U}|^{2}}{2}\right)\right] \\
& =\frac{q}{m}{ }^{\perp} \bar{E}(t) \cdot \bar{Y} n .
\end{aligned}
$$

It remains to establish the inequality (7). Multiplying (5) by $\left(1+\ln F^{\tau}\right)$, integrating with respect to $(\bar{X}, V)$, using (21) and passing to the limit for $\tau \searrow 0$, lead to

$$
\partial_{t} \int_{\mathbb{R}^{2}} \int_{\mathbb{R}^{3}} F \ln F \mathrm{~d} V \mathrm{~d} \bar{X}+\partial_{X_{3}} \int_{\mathbb{R}^{2}} \int_{\mathbb{R}^{3}} F V_{3} \ln F \mathrm{~d} V \mathrm{~d} \bar{X} \leq 0 .
$$

Taking into account that

$$
\ln F=\ln \frac{\omega^{2} n}{(2 \pi)^{5 / 2} \frac{\mu^{2} \theta^{3 / 2}}{\mu-\theta}}-\frac{|\bar{V}-\bar{U}|^{2}}{2 \frac{\mu \theta}{\mu-\theta}}-\frac{\left(V_{3}-U_{3}\right)^{2}}{2 \theta}-\frac{\left|\omega \bar{X}+{ }^{\perp} \bar{V}-\bar{Y}\right|^{2}}{2 \mu}
$$

we obtain

$$
\int_{\mathbb{R}^{2}} \int_{\mathbb{R}^{3}} F \ln F \mathrm{~d} V \mathrm{~d} \bar{X}=n \ln \frac{\omega^{2} n}{(2 \pi)^{5 / 2} \frac{\mu^{2} \theta^{3 / 2}}{\mu-\theta}}-\frac{5}{2} n
$$

and

$$
\int_{\mathbb{R}^{2}} \int_{\mathbb{R}^{3}} F V_{3} \ln F \mathrm{~d} V \mathrm{~d} \bar{X}=n U_{3} \ln \frac{\omega^{2} n}{(2 \pi)^{5 / 2} \frac{\mu^{2} \theta^{3 / 2}}{\mu-\theta}}-\frac{5}{2} n U_{3}
$$

implying that

$$
\partial_{t}\left[n \ln \frac{\omega^{2} n}{(2 \pi)^{5 / 2} \frac{\mu^{2} \theta^{3 / 2}}{\mu-\theta}}-\frac{5}{2} n\right]+\partial_{X_{3}}\left[n U_{3} \ln \frac{\omega^{2} n}{(2 \pi)^{5 / 2} \frac{\mu^{2} \theta^{3 / 2}}{\mu-\theta}}-\frac{5}{2} n U_{3}\right] \leq 0 .
$$

Combining to the continuity equation (6), one gets

$$
\partial_{t}\left(n \ln \frac{n}{\frac{\mu^{2} \theta^{3 / 2}}{\mu-\theta}}\right)+\partial_{X_{3}}\left(n U_{3} \ln \frac{n}{\frac{\mu^{2} \theta^{3 / 2}}{\mu-\theta}}\right) \leq 0, \quad\left(t, X_{3}\right) \in \mathbb{R}_{+} \times \mathbb{R} .
$$




\section{Acknowledgement}

This work has been carried out within the framework of the EUROfusion Consortium and has received funding from the Euratom research and training programme 20142018 under grant agreement No 633053. The views and opinions expressed herein do not necessarily reflect those of the European Commission.

\section{References}

[1] C. Bardos, F. Golse, D. Levermore, Fluid dynamic limits of kinetic equations. I. Formal derivations. J. Statist. Phys. 63(1991) 323-344.

[2] C. Bardos, F. Golse, D. Levermore, Fluid dynamic limits of kinetic equations. II. Convergence proofs for the Boltzmann equation. Comm. Pure Appl. Math. 46(1993) 667-753.

[3] M. Bostan, The Vlasov-Poisson system with strong external magnetic field. Finite Larmor radius regime, Asymptot. Anal., 61(2009) 91-123.

[4] M. Bostan, Transport equations with disparate advection fields. Application to the gyrokinetic models in plasma physics, J. Differential Equations 249(2010) 16201663.

[5] M. Bostan, On the Boltzmann equation for charged particle beams under the effect of strong magnetic fields, Discrete Contin. Dyn. Syst. Ser. B, Vol. 20, No. 2, pp. 339-371 (2015).

[6] M. Bostan, Multi-scale analysis for linear first order PDEs. The finite Larmor radius regime, SIAM J. Math. Anal., Vol. 48, No. 3, pp. 2133-2188 (2016).

[7] M. Bostan, High magnetic field equilibria for the Fokker-Planck-Landau equation, Ann. Inst. H. Poincaré Anal. Non Linéaire Vol. 33, No. 4, pp. 899-931 (2016).

[8] M. Bostan, C. Caldini-Queiros, Finite Larmor radius approximation for collisional magnetized plasmas, C. R. Acad. Sci. Paris, Ser. I Math. 350, pp. 879-884 (2012).

[9] M. Bostan, C. Caldini-Queiros, Finite Larmor radius approximation for collisional magnetic confinement. Part I: the linear Boltzmann equation, Quart. Appl. Math. Vol. LXXII(2014) 323-345.

[10] M. Bostan, C. Caldini-Queiros, Finite Larmor radius approximation for collisional magnetic confinement. Part II: the Fokker-Planck-Landau equation, Quart. Appl. Math. Vol. LXXII(2014) 513-548.

[11] M. Bostan, A. Finot, M. Hauray, The effective Vlasov-Poisson system for strongly magnetized plasmas, C. R. Acad. Sci. Paris, Ser. I 354(2016) 771-777.

[12] M. Bostan, A. Finot, The effective Vlasov-Poisson system for the finite Larmor radius regime, SIAM J. Multiscale Model. Simul. 14(2016) 1238-1275. 
[13] M. Bostan, I.M. Gamba, Impact of strong magnetic fields on collision mechanism for transport of charged particles, J. Stat. Phys., Vol. 148, No. 5, pp. 856-895 (2012).

[14] A.J. Brizard, Variational principle for non linear gyrokinetic Vlasov-Maxwell equations, Phys. Plasmas 7(2000) 4816-4822.

[15] A.J. Brizard, A guiding-center Fokker-Planck collision operator for nonuniform magnetic fields, Phys. Plasmas 11(2004) 4429-4438.

[16] A.J. Brizard, T.S. Hahm, Foundations of nonlinear gyrokinetic theory, Rev. Modern Phys. 79(2007) 421-468.

[17] C. Cercignani, R. Illner, M. Pulvirenti, The mathematical theory of dilute gases, Applied Mathematical Sciences, 106, Springer-Verlag New-York 1994.

[18] L. Desvillettes, C. Villani, On the spatially homogeneous Landau equation for hard potentials. I Existence, uniqueness and smoothness, Comm. Partial Differential Equations 25(2000) 179-259.

[19] L. Desvillettes, C. Villani, On the spatially homogeneous Landau equation for hard potentials. II H-theorem and applications, Comm. Partial Differential Equations $25(2000) 261-298$.

[20] L. Desvillettes, Plasma kinetic models: the Fokker-Planck-Landau equation, Modelling and computational methods for kinetic equations, 171-193, Model. Simul. Sci. Eng. Technol., Birkhäuser Boston, MA 2004.

[21] E. Frénod, E. Sonnendrücker, The finite Larmor radius approximation, SIAM J. Math. Anal. 32(2001) 1227-1247.

[22] X. Garbet, G. Dif-Pradalier, C. Nguyen, Y. Sarazin, V. Grandgirard, Ph. Ghendrih, Neoclassical equilibrium in gyrokinetic simulations, Phys, Plasmas 16(2009).

[23] D. Levermore, Entropic convergence and the linearized limit for the Boltzmann equation. Comm. Partial Differential Equations 18(1993) 1231-1248.

[24] D. Levermore, Moment closure hierarchies for kinetic theories. J. Statist. Phys. 83(1996) 1021-1065.

[25] X.Q. Xu, M.N. Rosenbluth, Numerical simulation of ion-temperature-gradientdriven modes, Phys. Fluids, B 3(1991) 627-643. 